\title{
Effect of temperature difference between channel walls on the heat transfer characteristics of nanoscale-confined gas
}

\author{
Reza Rabani ${ }^{\text {a }}$, Ghassem Heidarinejad ${ }^{\mathrm{a}, *}$, Jens Harting ${ }^{\text {b,c }}$, Ebrahim Shirani ${ }^{\mathrm{d}}$ \\ ${ }^{a}$ Faculty of Mechanical Engineering, Tarbiat Modares University, 14115143, Tehran, Iran \\ ${ }^{\mathrm{b}}$ Helmholtz Institute Erlangen-Nürnberg for Renewable Energy (IEK-11), Forschungszentrum Jülich, Fürther \\ Strasse 248, 90429 Nuremberg, Germany \\ ${ }^{c}$ Department of Applied Physics, Eindhoven University of Technology, PO box 513, 5600MB Eindhoven, The \\ Netherlands \\ ${ }^{d}$ Department of Mechanical Engineering, Foolad Institute of Technology, 8491663763, Fooladshahr, Isfahan, Iran \\ *Corresponding author. Tel.: +98 21 82883361; fax: +98 2188005040 \\ E-mail addresses: reza.rabani@modares.ac.ir (R.Rabani); gheidari@ modares.ac.ir, gheidari@ alum.mit.edu (G. \\ Heidarinejad*); j.harting@fz-juelich.de (J.Harting); eshirani@cc.iut.ac.ir, director@jafmonline.net (E.Shirani)
}

\begin{abstract}
We present molecular dynamics simulations of stationary argon gas in nanoscale confinement and under various temperature differences between walls. For a channel of $5.4 \mathrm{~nm}$ height, we vary the gas density and find that in addition to the temperature difference between the walls, the absolute temperature of each wall plays an important role in the determination of the gas molecule distribution regardless of the level of rarefaction. The combined effect of the wall force field, the temperature difference between the walls and the wall temperature leads to the fact that the normalized temperature profile along the channel height does not coincide for various temperature differences between the walls. As the gas density is increased, it is observed that the wall force field effect on the density and temperature profiles reduces considerably due to the increment in the magnitude of the gas force field for all implemented temperature differences. Considering the temperature profiles and the distribution of the effective local thermal conductivity (ELTC) along the channel height, it is inferred that a diffusive transport mechanism is dominant throughout the dense gas medium. Besides, as the gas becomes rarefied, ballistic transport in the bulk region and diffusive transport in the regions close to the walls are observed. Furthermore, the effective thermal conductivity is a function of the implemented temperature differences between the walls and its value at $300 \mathrm{~K}$ varies from 0.18 to $12 \mathrm{~mW} / \mathrm{mK}$ as the bulk gas density changes from 1.95 to $196 \mathrm{~kg} / \mathrm{m}^{3}$.
\end{abstract}

Keywords: Wall force field, Temperature profile, Heat transfer, Effective thermal conductivity, Ballistic transport mechanism, Diffusive transport mechanism

\section{Introduction}

Since nanoscale confined gas is widely encountered in micro/nanoelectromechanical systems (MEMS/NEMS), it is necessary to achieve a more profound understanding of its flow and heat transfer behavior in order to enhance the design and performance of such systems [1-3]. One of the most interesting properties of the nanoscale-confined gas in nanochannels is the large surface/volume ratio, which may lead to opportunities to study new physical phenomena 
previously inaccessible using large-scale devices. The local Knudsen number $(K n=\lambda / H$, where $\lambda$ is the local gas mean free path and $H$ is the characteristic length of the domain) is an indicator of rarefaction and the relevance of non-equilibrium effects of the medium. While these phenomena can be studied for example by kinetic theory based methods such as a direct solution of the Boltzmann transport equation [4], the surface/gas interactions ("surface force field") and the surface adsorption which arises under these conditions require a molecular level of simulation to be analyzed exactly. The molecular dynamics method is a computer simulation technique that solves Newton's equations of motion for particles (atoms and molecules) and their interactions. It is one of the most widely used methods that enables an adequate resolution of the molecular structure of gases and surfaces as well as their interactions at atomic length and time scales.

The known accumulation of gas molecules near the boundary and the resulting difference in the velocity profile from the prediction obtained from a continuum description can be observed [5-7]. Considering the fact that the gas state evolves through intermolecular collisions, the channel dimension in periodic directions should extend at least for one mean free path. For high Knudsen number gas states, the periodic dimension leads to a need for modeling a relatively large number of gas molecules and an extremely large number of wall molecules. Therefore, the "smart wall molecular dynamics" (SWMD) model was proposed and implemented in the molecular dynamics simulation which reduces the CPU time drastically. SWMD is a cold wall model that represents three-dimensional FCC walls using only 74 wall molecules which are kept in the memory and utilized for each collision of a gas molecule with the surface [8,9].

In a series of studies, Barisik and Beskok investigated the effect of the wall force field on different gas properties such as density, velocity, pressure and stress distribution in nanochannels by implementing the SWMD [8-14]. It was observed that besides an anisotropic variation of the shear and normal stresses in the near-wall region, significant variations in the gas velocity and density in this region are present $[9,10]$. Furthermore, it was shown that while the gas-wall interaction parameter influences the surface adsorption of the gas molecules, the transport in the bulk and near-wall regions of the nanochannels is determined by the Knudsen number and the gaswall interaction parameter [10-12]. Regardless of the molecular surface forces and adsorption of gas molecules to the walls, it was observed that the mean free path of the gas stays unaffected by the nanoconfinement. This observation is in accordance with kinetic theory [12].

It should be mentioned that due to the cold wall nature of the SWMD, there was no heat transfer between the gas and the walls present in the above-mentioned research and the heat was removed by applying a thermostat on the gas. However, in various applications, the heat transfer between gas and walls is considerable and cannot be neglected. As an example, the slider and the disk in hard disk drives are approximately $2 \sim 5 \mathrm{~nm}$ apart. At such small distances, they might experience a temperature difference of $20 \sim 400 \mathrm{~K}$ [15-17]. In addition, the heat transfer mechanism is unclear if the wall force field covers a notable portion of the channel height. It is known from the literature [18] that diffusive transport in the gas medium dominates when $K n \ll 1$ in a way that heat is transferred between the channel walls by intermolecular gas collisions. In contrast, it is observed that ballistic heat transfer dominates as $K n \gg 1$ since the heat is transferred by direct collisions of 
gas molecules with the walls. The heat transfer mechanism between these two limiting conditions in the presence of the wall force field has not been studied in detail to the best of our knowledge, yet. A literature review reveals that while the heat transfer in liquid confined nanoscale media has been studied extensively by using appropriate wall/liquid interaction potentials, the heat transfer in nanoscale-confined gas has not been studied as well in details [19,20,29-36,21-28]. This might be due to the fact that the simulation of a nanoscale confined gas requires a very large dimension in the periodic direction which leads to an enormous number of wall molecules in comparison with a nanoscale confined liquid simulation. Our earlier study was focused on the heat transfer characteristic of static argon gas within nanoscale-confinement for wide range of Knudsen number corresponds to the transition regime. While temperature difference of $20 \mathrm{~K}$ was applied on the channel walls, changing the gas density and the channel height lead to different modified Knudsen numbers $(k=(\sqrt{\pi} / 2) K n)$ in the range of 0.1 to 10 . The main reason behind choosing $k$ instead of $K n$ refers to the fact that our research is based on the previous studies [9-14] in which nanoconfinement effects on the velocity, density and stress distribution are discussed thoroughly. In those papers, the modified Knudsen number was used and all the discussions were based on this. Since in our current study the channel dimensions and Knudsen number are of the same order as in the above-mentioned studies, we have decided to use the modified Knudsen number in order to assure consistency with the previous works.

It was observed that as the gas density is increased for constant channel height, a reduction in the maximum normalized gas density near the walls occurs which leads to a higher temperature jump in comparison with the case with the same Knudsen number but when the channel height is increased and the gas density is kept constant. Meanwhile, it was shown that for all Knudsen numbers in the transition regime, the wall force field and the interfacial thermal resistance form a notable portion of the total thermal resistance even for near micrometer-sized (540 nm height) channels. In addition, it was found that while the wall force field reduces the local thermal conductivity in the near-wall in comparison with the bulk region, the local thermal conductivity in this region strongly depends on the gas density [37].

In this paper, we intend to explore the distribution of the density and temperature, the heat flux and the thermal resistance of nanoscale confined argon gas as a function of the temperature difference between the channel walls. In addition, we determine the heat transfer mechanism for finite Knudsen numbers in the presence of the wall force field. To address these, we perform different sets of molecular dynamics simulations at $k=10,1$ and 0.1 for temperature differences of $0,30,100,200$ and $300 \mathrm{~K}$ between the channel walls. A temperature difference of $0 \mathrm{~K}$ corresponds to the isothermal condition between the gas and the walls. In all cases, the channel height is considered to be $\mathrm{H}=5.4 \mathrm{~nm}$ and the wall force field covers approximately $40 \%$ of the channel height. The variation of the modified Knudsen number is achieved by changing the gas density. This manuscript is organized as follows: In Section 2, we describe the setup of our molecular dynamics simulations. In Section 3, we discuss our simulation results and in particular the combined effect of the temperature difference and the wall force field on the distribution of the gas properties and heat transfer mechanism. Finally, we conclude in Section 4. 


\section{Numerical method}

We simulate a gas of argon atoms confined between two parallel plates that are a distance $H=$ $5.4 \mathrm{~nm}$ apart by using LAMMPS (Large-Scale Atomic/Molecular Massively Parallel Simulator) from Sandia National Laboratories [38]. Periodic boundary conditions are applied in the axial (x) and lateral $(\mathrm{z})$ directions. The truncated (6-12) Lennard-Jones ( $\mathrm{L}-\mathrm{J})$ potential is used to model the van der Waals interactions between different atoms:

$$
\phi(r)=4 \varepsilon\left(\left(\left(\frac{\sigma}{r_{i j}}\right)^{12}-\left(\frac{\sigma}{r_{i j}}\right)^{6}\right)-\left(\left(\frac{\sigma}{r_{c}}\right)^{12}-\left(\frac{\sigma}{r_{c}}\right)^{6}\right)\right)
$$

where $r_{i j}$ is the interatomic distance, $r_{c}$ is the cutoff distance and $\varnothing\left(r_{C}\right)$ is the value of the interatomic potential at $r=r_{C}$. The depth of the potential well for argon is $\varepsilon=119.8 \times k_{b}$, where the Boltzmann constant is $k_{b}=1.3806 \times 10^{-23} \mathrm{~J} / \mathrm{K}$, the molecular diameter is $\sigma=0.3405 \mathrm{~nm}$ and the mass of an argon atom is $m=6.63 \times 10^{-26} \mathrm{~kg}$. Considering the fact that the L-J potential is negligible at large molecular distance, the cut off radius is commonly set to $r_{C}=1.08 \mathrm{~nm}$ for a dilute gas [25]. For the wall atoms, the same parameters as for argon are used $\left(m_{\mathrm{wall}}=m_{\mathrm{Ar}}\right.$, $\sigma_{\text {wall }}=\sigma_{\mathrm{Ar}}$ ) and the strength of the gas-wall interactions is equal to that of the gas-gas interactions $\left(\varepsilon_{\text {wall-Ar }}=\varepsilon_{\mathrm{Ar}-\mathrm{Ar}}\right)$. Two layers of FCC (face-centered cubic) structured atoms are used to simulate the walls. We apply a Verlet algorithm to integrate Newton's second law [39]. The "interactive thermal wall model" (ITWM) is used to control the wall temperature [40], where the wall particles are linked to each other by springs. They are fixed onto their initial lattice positions and oscillate around their equilibrium position. Therefore, they are able to exchange momentum and energy with the fluid particles. On each layer of wall molecules, a velocity-scaling thermostat is applied in order to obtain a uniform temperature distribution in the walls. In this way, the heat is properly transferred to/from the gas through the walls and there is no need to apply any thermostat on the gas anymore [40,41]. In the present study, the value of $K_{S}=500 \varepsilon \sigma^{-2}$ is used as wall stiffness that determines the strength of the bonds between the wall particles [42]. Then, the total force experienced by the wall and fluid atoms is [40]

$$
\begin{aligned}
& F_{\text {wall }}\left(r_{i}\right)=\sum_{j=1}^{N_{f}} \frac{\partial \phi\left(r_{i j}\right)_{\text {fluid-wall }}}{\partial r_{i j}}+K_{S}\left(\left|r_{0}-r_{i}\right|\right), \\
& F_{\text {fluid }}\left(r_{i}\right)=\sum_{j=1}^{N_{w}} \frac{\partial \phi\left(r_{i j}\right)_{\text {fluid-wall }}}{\partial r_{i j}}+\sum_{j=1}^{N_{f}} \frac{\partial \phi\left(r_{i j}\right)_{\text {fluid-fluid }}}{\partial r_{i j}},
\end{aligned}
$$

where $N_{f}$ and $N_{w}$ are the numbers of fluid and solid atoms in the volume given by the cutoff radius of each atom and $\left|r_{0}-r_{i}\right|$ is the distance between the current position of the wall atom and the original lattice positions. Considering $T_{H}$ and $T_{C}$ to be the temperature of the top and bottom wall, respectively, the temperature difference between the walls is defined as $\Delta T=T_{H}-T_{C}$ and the mean temperature of the system is defined as $T_{\text {mean }}=\left(T_{H}+T_{C}\right) / 2$. A Maxwell-Boltzmann velocity distribution with the mean temperature is used to initialize the argon and wall atoms at 
the beginning of the simulation. To reach thermal equilibrium, we let the initial particle distribution evolve for $2 \times 10^{6}$ time steps ( $8 \mathrm{~ns}$ ) using a timestep of 4 fs by applying a temperature-rescaling thermostat and the NVE ensemble to the wall and gas atoms. After that, the temperature of the walls is changed to the desired ones allowing the system to relax towards the expected temperature difference in $n_{s s c}$ time steps. Finally, $n_{\text {ave }}$ timesteps are performed to average microscopic quantities in order to obtain macroscopic properties of the gas. Longer time averaging is also performed in each case to confirm the convergence of quantities such as the density and temperature profiles. The channel height is determined from the centerlines of the first layer of wall molecules at the top and bottom surfaces. The computational domain is divided into bins of approximately $0.108 \mathrm{~nm}$ and $0.054 \mathrm{~nm}$ for the density and temperature, respectively in the perpendicular direction to the walls. In order to show the density and temperature profile in a similar way, only half of the data of the density profiles is shown in all figures. To be able to compare the effect of the wall force field on various temperature differences between the walls, the non-dimensionalized temperature profile is presented by

$$
T_{(i)}=\left(T_{(i)}-T_{\text {mean }}\right) /(\Delta T / 2) \text {, }
$$

where $T_{i}$ is the gas temperature in ith bin. In this way for all cases, the values of -1 and 1 are assigned to $T_{C}$ and $T_{H}$, respectively, and the gas temperature profile is scaled between these values accordingly. For the same reason, the density is normalized in each case by dividing the gas density in each bin by the gas density in the bin at the center of the channel. Figure 1 depicts a schematics sketch of the simulation domain and the averaging bin.

Different temperatures for walls lead to a thermal gradient in the perpendicular direction and consequently, this gradient generates a heat flux between the parallel walls. The heat flux vector is determined using the Irving-Kirkwood $(\mathrm{I}-\mathrm{K})$ expression $[43,44]$ :

$$
\begin{aligned}
& J_{l}=\frac{1}{\operatorname{Vol}}\left\langle\sum_{i}^{N_{f}} V_{l}^{i}\left(E^{i}+\emptyset^{i}\right)+\frac{1}{2} \sum_{i, j}^{N_{f}} Z^{i, j} r_{l}^{i j}\right\rangle, \\
& Z^{i, j}=f^{i j} \cdot V^{i}, \\
& E^{i}=\frac{1}{2} m^{i}\left(\left(V_{x}^{i}\right)^{2}+\left(V_{y}^{i}\right)^{2}+\left(V_{z}^{i}\right)^{2}\right) .
\end{aligned}
$$

Considering $l$ as the axes of the Cartesian coordinate system, $V_{l}^{i}$ is the velocity component of particle $i$ in $l$-direction. Furthermore, $E^{i}$ is the kinetic energy of particle $i$ and $r_{l}^{i j}=r_{l}^{j}-r_{l}^{i}$ is the $l$ th component of the relative distance vector between particle $i$ and $j$. Additionally, $f^{i j}$ is the vector of intermolecular force exerted on particle $i$ by particle $j$ and $V^{i}$ is the velocity vector. To improve the quality of our data, every measurement is obtained from averaging over the outcome from three independent simulations. It should be noticed that the above-mentioned formula only accounts for the conduction heat transfer between the channel walls. The radiative heat flux between the walls is not considered in our analysis since it does not affect the gas temperature and does not change the effective thermal conductivity of the gas medium. A snapshot of the simulation domain with the initial distribution of the wall and gas atoms is displayed in Fig. 2. 


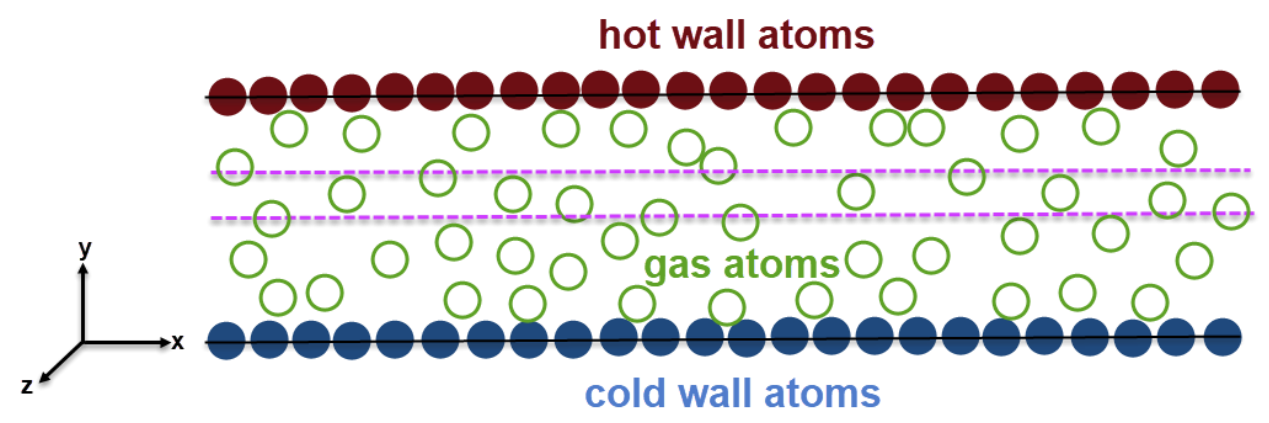

Fig. 1 Schematic sketch of domain and averaging bin (dashed lines)

In all 3D simulations, each wall is constructed using 101003 atoms which contribute drastically to the computational time required. The simulation domain has the dimension of $54 \times$ $5.4 \times 54 \mathrm{~nm}$ and the gas density varies in such a way that the modified Knudsen number is 10,1 or 0.1 . $k=10$ and $k=1$ correspond to rarefied gases, and the cut off radius for all interactions is kept at $r_{c}=1.08 \mathrm{~nm}$. For $k=0.1$, the gas becomes dense and $r_{c}=2.7 \mathrm{~nm}$ is used for the cutoff radius [9]. Indeed, due to the temperature of each surface, the distribution of gas atoms near each wall is different which changes the gas density in the middle of the channel (bulk region). Thus, the number of argon atoms in each case is determined in a way that the gas density in the bulk region corresponds to the desired Knudsen number. The exact value of the number of the argon atoms, $n_{s s c}, n_{a v r}, T_{H}, T_{C}$ and domain specification of each case are presented in the corresponding Table $[9,10]$.

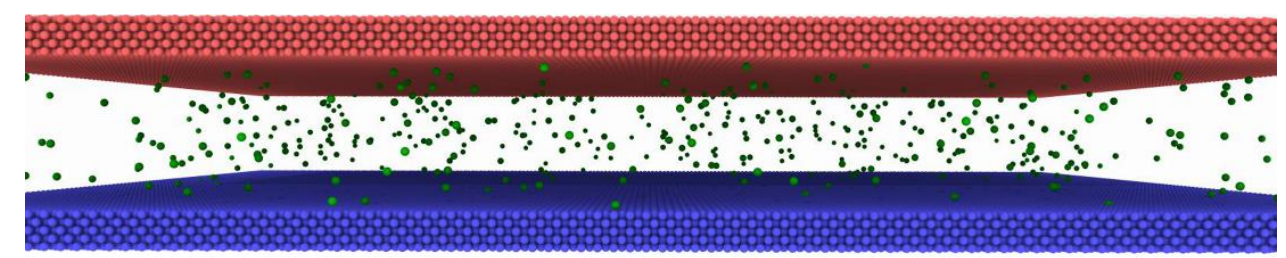

(a) $k=10$

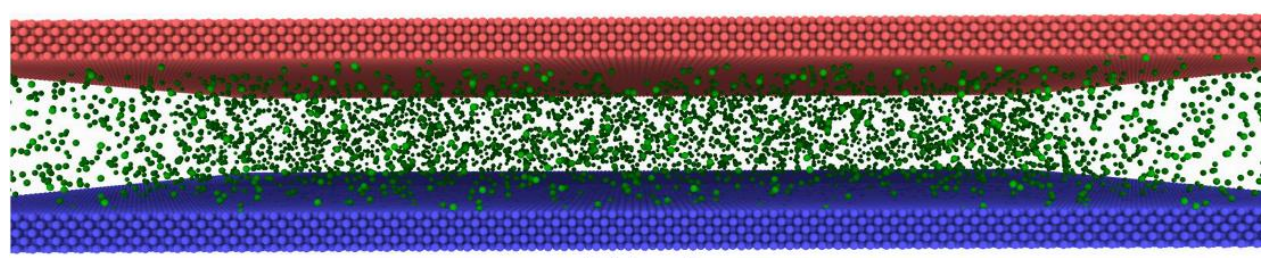

(b) $k=1$

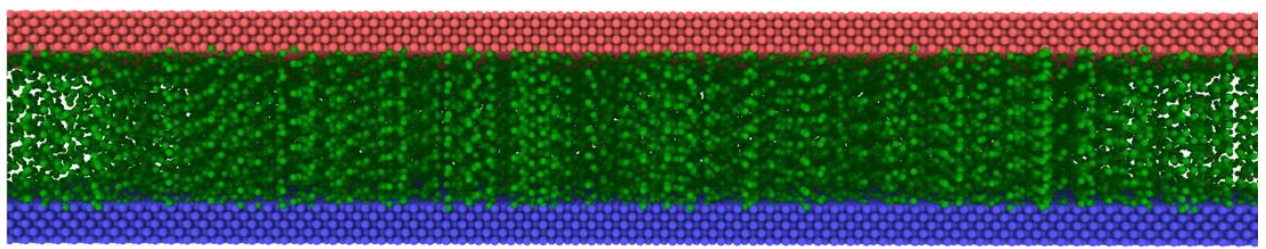

(c) $\mathrm{k}=0.1$

Fig. 2 Snapshot of the simulation domain for $k=10(\mathbf{a}), k=1(\mathbf{b})$ and $k=0.1(\mathbf{c})$ 


\section{Results and discussion}

We start our investigation by applying isothermal conditions on the walls and the gas medium. For $k=10,1$ and 0.1 , a temperature of $300,400,500$ and $600 \mathrm{~K}$ is applied on the walls and the gas initially. Due to the thermostat on the walls, the walls are kept at the prescribed temperature and the gas evolves to the desired temperature through gas-wall collisions. The simulation parameters are summarized in Table 1. The distribution of the gas temperature and density along the height of the channel are shown in Fig. 3 for the three Knudsen numbers. The distribution of the gas density in Figs. 3a, c and e reveal that regardless of the wall temperature, the maximum value of the density can be found approximately $0.2 \mathrm{~nm}$ from each wall. This refers to the wall force field region which extends at approximately $1 \sim 2 \mathrm{~nm}$ from each wall and changes the fluid distribution in this region. Indeed, the wall force field increases the residence time of the gas molecules that enter its penetration depth and this causes the accumulation of gas molecules near each wall. This observation is in accordance with SWMD studies investigating the effect of the wall force field on the distribution of gas atoms [8-14]. It is interesting to note that for all three Knudsen numbers, the distribution of the gas molecules along the channel height not only depends on the wall force field but also on the temperature of the medium. For $k=10$, we observe that the maximum value of the gas density near each wall decreases from 5.77 to $3.25 \mathrm{~kg} / \mathrm{m}^{3}$ as the mean temperature increases from 300 to $600 \mathrm{~K}$.

Table 1 MD simulation details for various modified Knudsen numbers under isothermal conditions

\begin{tabular}{|c|c|c|c|c|c|c|}
\hline Case & $T$ & $n_{s s c}$ & $n_{\text {ave }}$ & $r_{\mathrm{c}}(\mathrm{nm})$ & \# atoms & $\rho_{\text {bulk }}\left(\mathrm{kg} / \mathrm{m}^{3}\right)$ \\
\hline \multirow{4}{*}{$k \sim 10$} & 300 & $1 \times 10^{6}$ & $2 \times 10^{6}$ & 1.08 & 480 & 1.94 \\
\hline & 400 & $1 \times 10^{6}$ & $2 \times 10^{6}$ & 1.08 & 480 & 1.97 \\
\hline & 500 & $1 \times 10^{6}$ & $2 \times 10^{6}$ & 1.08 & 480 & 2.05 \\
\hline & 600 & $1 \times 10^{6}$ & $2 \times 10^{6}$ & 1.08 & 480 & 2.07 \\
\hline \multirow{4}{*}{$k \sim 1$} & 300 & $1 \times 10^{6}$ & $1.5 \times 10^{6}$ & 1.08 & 4800 & 19.3 \\
\hline & 400 & $1 \times 10^{6}$ & $1.5 \times 10^{6}$ & 1.08 & 4800 & 19.7 \\
\hline & 500 & $1 \times 10^{6}$ & $1.5 \times 10^{6}$ & 1.08 & 4800 & 20.2 \\
\hline & 600 & $1 \times 10^{6}$ & $1.5 \times 10^{6}$ & 1.08 & 4800 & 20.6 \\
\hline \multirow{4}{*}{$k \sim 0.1$} & 300 & $1 \times 10^{6}$ & $1 \times 10^{6}$ & 2.7 & 48000 & 197 \\
\hline & 400 & $1 \times 10^{6}$ & $1 \times 10^{6}$ & 2.7 & 48000 & 202 \\
\hline & 500 & $1 \times 10^{6}$ & $1 \times 10^{6}$ & 2.7 & 48000 & 205 \\
\hline & 600 & $1 \times 10^{6}$ & $1 \times 10^{6}$ & 2.7 & 48000 & 208 \\
\hline
\end{tabular}


(a)

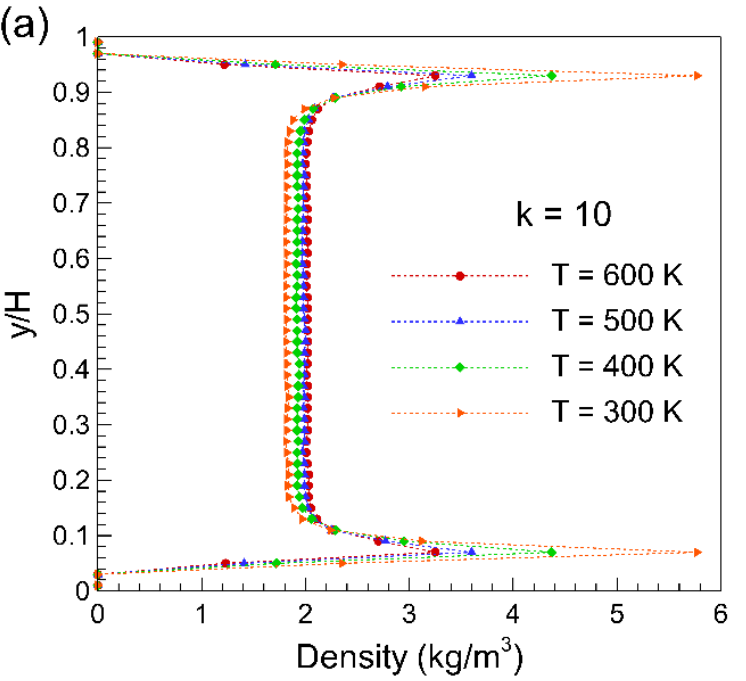

(c)

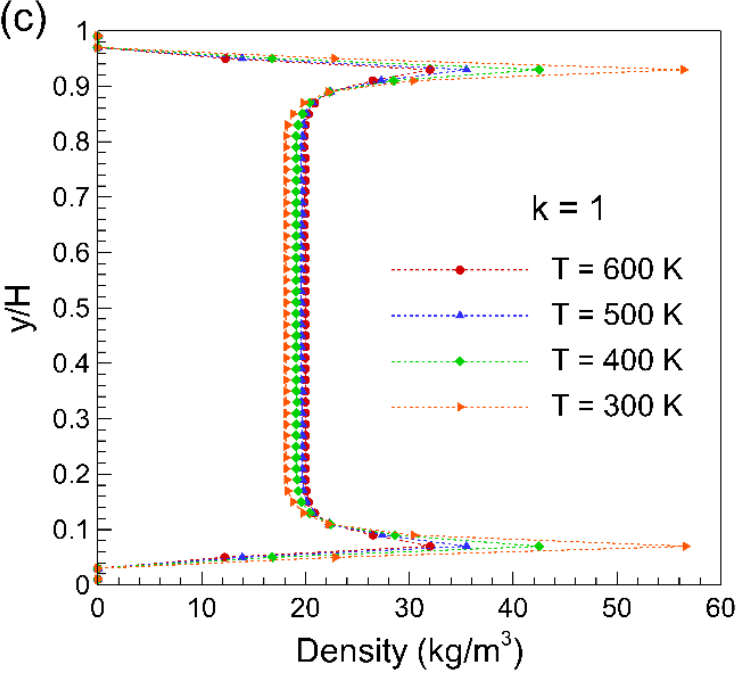

(e)

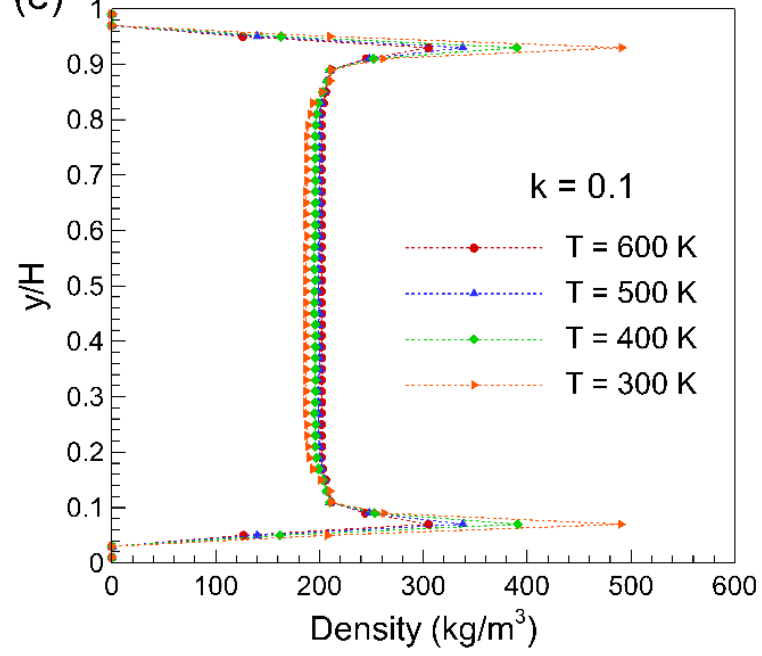

(b)

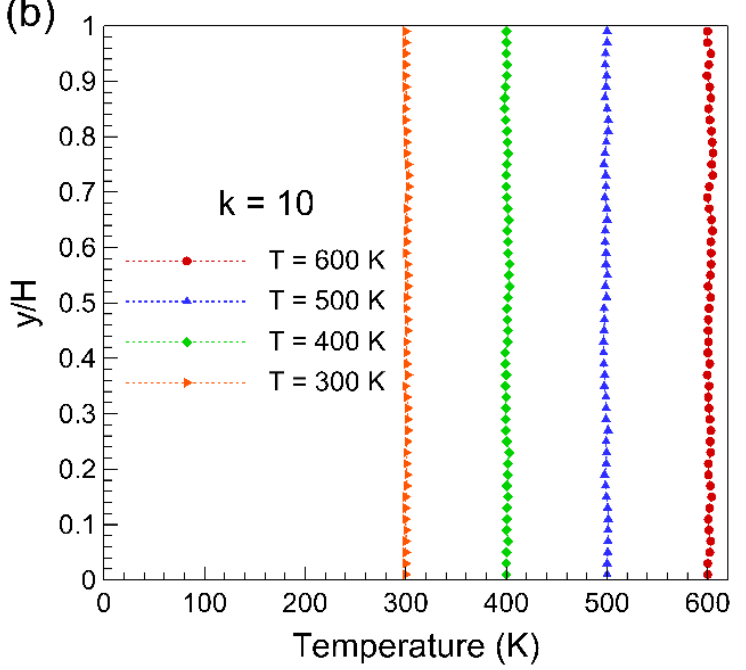

(d)

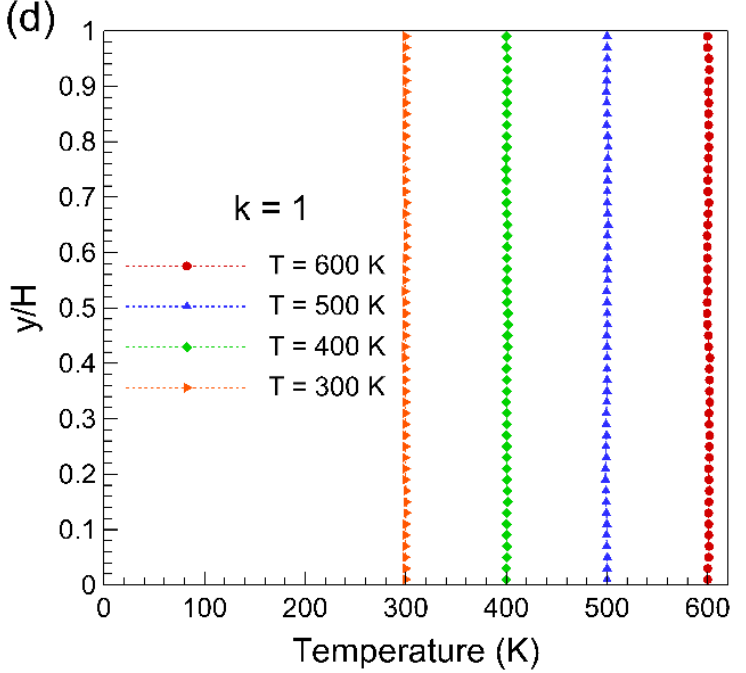

(f)

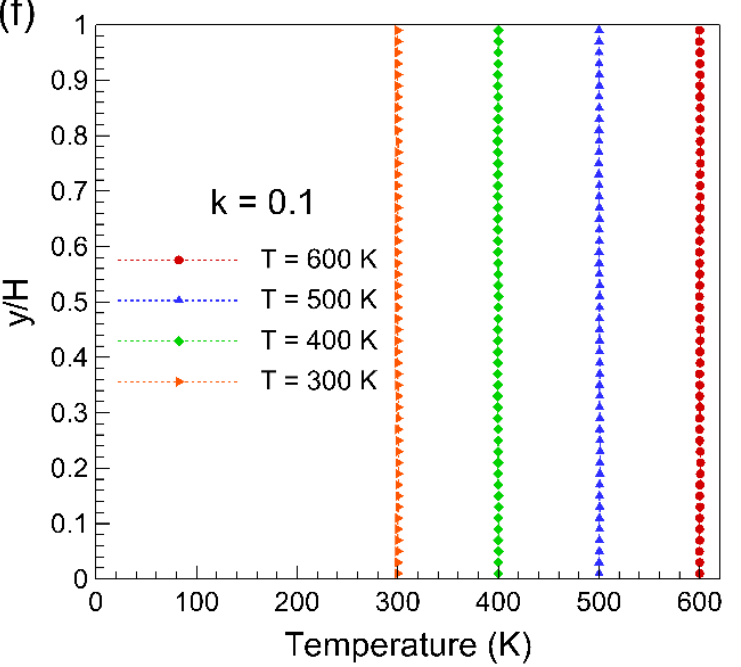

Fig. 3 Distribution of the gas density and temperature along the channel height for isothermal conditions between the walls and the gas for three modified Knudsen numbers 
Actually, as the temperature of the gas and the walls increases, the energy of the impinging gas atoms enhances notably allowing them to leave the wall force field region more quickly. As a direct consequence, the gas accumulation in the wall force field region decreases. The reduction in the maximum gas density near each wall leads to an increased density of the gas in the bulk region as it is shown in Fig. 3 and Table 1. As an example, for $k=10$, while the mean temperature is increased from 300 to $600 \mathrm{~K}$, the bulk gas density increases from 1.94 to $2.07 \mathrm{~kg} / \mathrm{m}^{3}$, consequently. The decrease in the maximum gas density near each wall and therefore, the increase of the gas density in the bulk region is also observed for $k=1$ and $k=0.1$. The distribution of the gas temperature in the channel can be observed in Figs. 3b, $\mathrm{d}$ and $\mathrm{f}$ for the three Knudsen numbers. According to these figures, the uniform temperature distribution in the gas along the channel height depicts the validity of the prescribed method to obtain the desired gas temperature.

It should be noted that in our study, the modified Knudsen number is defined based on the gas density and its corresponding mean free path in the middle of the channel. This is in accordance with the way that the Knudsen number is defined for the nanoconfined gas medium in the literature [8-14]. Table 1 clearly depicts that as the temperature differences between the walls are increased in a Knudsen number, the bulk gas density is changed. This fact was considered in defining the Knudsen number through the manuscript. As the bulk gas density changes notably, the number of gas atoms was varied accordingly so we had approximately a constant gas density ( constant mean free path) in the middle of the channel for various temperature differences between the walls. The exact number of gas atoms for each case is depicted in its corresponding table and was obtained through the trial and error method. Reducing and increasing the total number of gas atoms in order to keep constant mean free path in the middle of the channel is a common method for keeping a constant Knudsen number in nanoconfinement gas medium in the literature [14].

Next, the effect of changing the temperature difference between the walls on the variation of different properties such as the normalized temperature and density profile, heat flux, thermal conductivity and thermal resistance of the gas medium are studied. In order to investigate the effect of the wall temperature on the distribution of the argon gas atoms in the vicinity of each wall, two sets of simulations were performed for each specified temperature difference. In the first set of simulations (case A), the base temperature is considered as $300 \mathrm{~K}$ and $T_{H}$ and $T_{C}$ are defined as $300+(\Delta T / 2) \mathrm{K}$ and $300-(\Delta T / 2) \mathrm{K}$, respectively. In the second set of simulations (case B), the base temperature is also considered as $300 \mathrm{~K}$ which was assigned to the bottom wall. $T_{H}$ and $T_{C}$ are then defined as $300+(\Delta T) \mathrm{K}$ and $300 \mathrm{~K}$, respectively. In this way for each case we have two sets of simulations where the temperature differences between the walls are the same while the temperature of the walls are different. In addition to the previously measured properties, this allows us to investigate the dependency of the gas atom distribution on the wall temperature.

Table 2 summarizes the simulation parameters for $k=10,1$ and 0.1 . Table 2 depicts that for the case A, in order to keep a constant Knudsen number in the middle of the channel, we need to increase the number of argon atoms as the temperature difference between the walls is increased. As an example, for $\Delta T=30 \mathrm{~K}, 480$ argon gas atoms result in $k=10$, while for a temperature difference of $300 \mathrm{~K}$, it increases to 580 . Figure 4 depicts the reason for such behavior. 
Table 2 MD simulation parameters for various temperature differences between walls

\begin{tabular}{|c|c|c|c|c|c|c|c|c|c|c|}
\hline$k$ & Case & $\Delta T$ & $T_{H}(\mathrm{~K})$ & $T_{C}(\mathrm{~K})$ & $n_{s S c}$ & $n_{\text {ave }}$ & \# atoms & $\rho_{\text {bulk }}\left(\frac{\mathrm{kg}}{\mathrm{m}^{3}}\right)$ & $k=\frac{\sqrt{\pi}}{2} K n$ & $k_{C}\left(\frac{m W}{m K}\right)$ \\
\hline \multirow{8}{*}{10} & \multirow{4}{*}{ A } & 30 & 315 & 285 & $10 \times 10^{6}$ & $50 \times 10^{6}$ & 480 & 1.95 & 10.82 & 17.7 \\
\hline & & 100 & 350 & 250 & $5 \times 10^{6}$ & $20 \times 10^{6}$ & 490 & 1.96 & 10.77 & 17.7 \\
\hline & & 200 & 400 & 200 & $3 \times 10^{6}$ & $10 \times 10^{6}$ & 500 & 1.98 & 10.66 & 17.7 \\
\hline & & 300 & 450 & 150 & $2 \times 10^{6}$ & $5 \times 10^{6}$ & 580 & 2.01 & 10.50 & 17.7 \\
\hline & \multirow{4}{*}{ B } & 30 & 330 & 300 & $10 \times 10^{6}$ & $50 \times 10^{6}$ & 480 & 1.98 & 10.66 & 18.2 \\
\hline & & 100 & 400 & 300 & $5 \times 10^{6}$ & $20 \times 10^{6}$ & 480 & 1.99 & 10.60 & 20.1 \\
\hline & & 200 & 500 & 300 & $3 \times 10^{6}$ & $10 \times 10^{6}$ & 480 & 2.03 & 10.39 & 22.4 \\
\hline & & 300 & 600 & 300 & $2 \times 10^{6}$ & $5 \times 10^{6}$ & 480 & 2.05 & 10.29 & 24.5 \\
\hline \multirow{8}{*}{1} & \multirow{4}{*}{ A } & 30 & 315 & 285 & $5 \times 10^{6}$ & $15 \times 10^{6}$ & 4800 & 19.9 & 1.06 & 18 \\
\hline & & 100 & 350 & 250 & $3 \times 10^{6}$ & $10 \times 10^{6}$ & 4850 & 19.9 & 1.06 & 18 \\
\hline & & 200 & 400 & 200 & $2 \times 10^{6}$ & $5 \times 10^{6}$ & 5000 & 19.7 & 1.07 & 18 \\
\hline & & 300 & 450 & 150 & $1 \times 10^{6}$ & $4 \times 10^{6}$ & 5800 & 19.9 & 1.06 & 18 \\
\hline & \multirow{4}{*}{ B } & 30 & 330 & 300 & $5 \times 10^{6}$ & $15 \times 10^{6}$ & 4800 & 19.8 & 1.06 & 18.8 \\
\hline & & 100 & 400 & 300 & $3 \times 10^{6}$ & $10 \times 10^{6}$ & 4800 & 20 & 1.05 & 20.5 \\
\hline & & 200 & 500 & 300 & $2 \times 10^{6}$ & $5 \times 10^{6}$ & 4800 & 20.3 & 1.03 & 22.8 \\
\hline & & 300 & 600 & 300 & $1 \times 10^{6}$ & $4 \times 10^{6}$ & 4800 & 20.2 & 1.04 & 24.9 \\
\hline \multirow{8}{*}{0.1} & \multirow{4}{*}{ A } & 30 & 315 & 285 & $1 \times 10^{6}$ & $2 \times 10^{6}$ & 48000 & 196 & 0.107 & 22.84 \\
\hline & & 100 & 350 & 250 & $1 \times 10^{6}$ & $2 \times 10^{6}$ & 48500 & 193 & 0.109 & 22.75 \\
\hline & & 200 & 400 & 200 & $1 \times 10^{6}$ & $2 \times 10^{6}$ & 52000 & 192 & 0.109 & 22.58 \\
\hline & & 300 & 450 & 150 & $1 \times 10^{6}$ & $2 \times 10^{6}$ & 67000 & 197 & 0.107 & 22.68 \\
\hline & \multirow{4}{*}{ B } & 30 & 330 & 300 & $1 \times 10^{6}$ & $2 \times 10^{6}$ & 48000 & 200 & 0.105 & 23.68 \\
\hline & & 100 & 400 & 300 & $1 \times 10^{6}$ & $2 \times 10^{6}$ & 48000 & 202 & 0.104 & 25.5 \\
\hline & & 200 & 500 & 300 & $1 \times 10^{6}$ & $2 \times 10^{6}$ & 48000 & 203 & 0.103 & 28 \\
\hline & & 300 & 600 & 300 & $1 \times 10^{6}$ & $2 \times 10^{6}$ & 48000 & 203 & 0.103 & 30.3 \\
\hline
\end{tabular}


Figures $4 \mathrm{a}$ and $\mathrm{b}$ show the distribution of normalized gas density and temperature for various temperature differences along the height of the channel for $k=10$, respectively. As discussed before, the maximum value of the density appears approximately $0.2 \mathrm{~nm}$ from each wall due to the presence of the wall force field. As a direct consequence of this distribution, the maximum and minimum temperature of the gas near the hot and cold wall occur in the wall force field region regardless of the implemented temperature differences as it is shown in Fig. 4b. The accumulation of gas atoms near each wall makes them collide with the walls much more frequently and the heat transfer between the walls and the gas increases notably which results in a closer temperature of the gas to the wall temperature in this region. Furthermore, it is clear from Fig. 4a that for small temperature differences in the order of $30 \mathrm{~K}$, the maximum of the normalized density in the nearwall region is about 3.46 and 3.20 times higher than the bulk density near the cold and hot walls, respectively. This shows that since the temperature difference is small and the temperature of the walls are in the same range, the maximum values of the normalized density are in the same order. As the temperature difference was increased in this case, the bottom wall becomes colder (up to $150 \mathrm{~K}$ ) and the upper wall becomes hotter (up to $450 \mathrm{~K}$ ). Figure 4a shows that in this situation the maximum gas densities near the cold and hot walls are about 11.2 and 2.13 times greater than the bulk density, respectively. It is clear that in comparison with $\Delta T=30 \mathrm{~K}$, the normalized gas density near the hot wall reduces by about 33\%, while near the cold wall, it increases by $323 \%$. These unequal changes in density near the hot and cold walls lead to a reduction of gas molecules in the bulk region. Therefore, we have to increase the number of argon atoms in order to keep the same Knudsen number in the bulk region as shown in Table 2. In addition, a higher temperature at the upper wall leads to an increase in the energy of impinging gas molecules.

Consequently, the residence time of gas molecules in the wall force region decreases resulting in a decreased density in this region as it is shown in Fig. 4a. However, it reduces the lower wall temperature so when the argon atoms hit the cold wall, they lose more energy and it takes more time for them to escape from the wall force field toward the bulk region. Therefore, the residence time in the wall force field region increases and the gas accumulates near the walls resulting in an increased density. Figure $4 \mathrm{a}$ shows that the distribution of the normalized gas temperature is a function of the temperature difference between the walls. It is expected that using equation (4) to normalize the temperature profile leads to a unique profile in the gas along with the channel height regardless of the temperature difference between the walls. Considering the density distribution of the gas along the channel height, the behavior of the normalized temperature profile in Fig. $4 \mathrm{~b}$ can be described. Indeed, changing the temperature difference between the walls from $30 \mathrm{~K}$ to $300 \mathrm{~K}$, increases the gas density near the cold wall. Thus, the temperature of the gas in the wall force field region becomes closer to the wall temperature. Simultaneously, it causes a decrease of the gas density near the hot wall so for the upper wall, the gas molecules cannot transfer heat as efficiently and this leads to a higher difference between the wall temperature and the gas. The combination of these two phenomena leads to a shift in the normalized temperature profile of the gas toward the left of the chart as the wall temperature difference increases. 
(a)

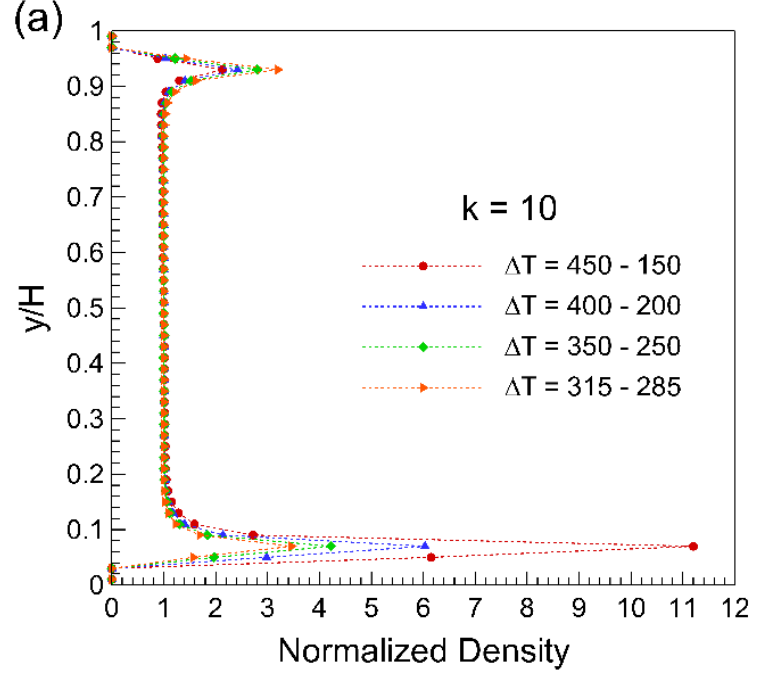

(c)

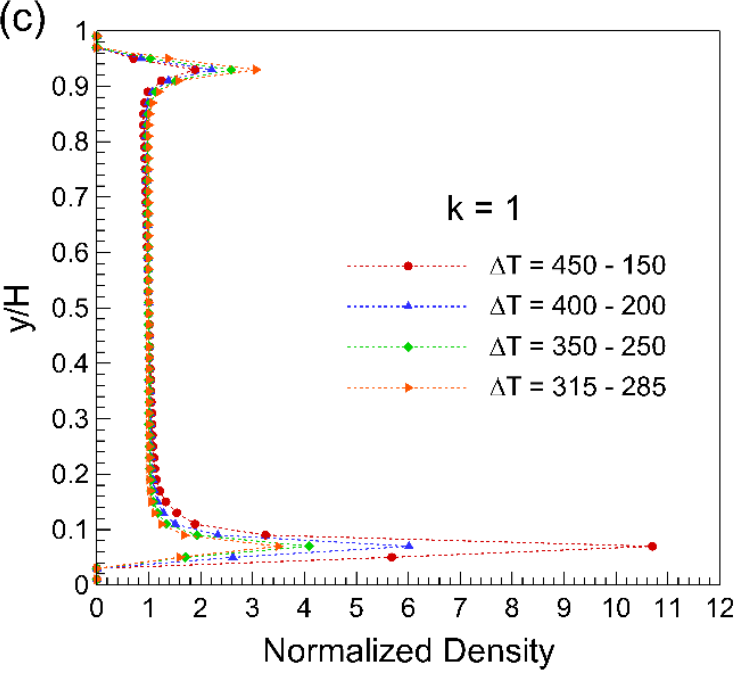

(e)

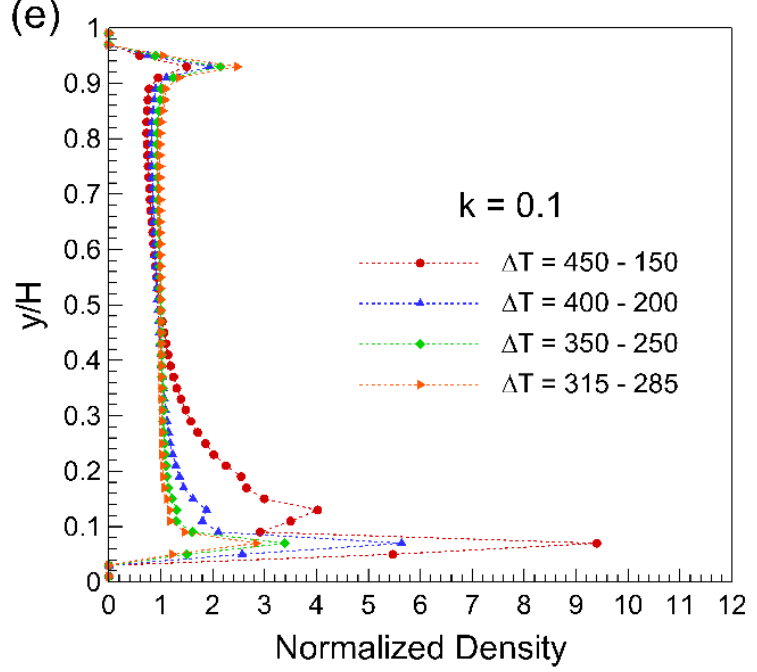

(b)

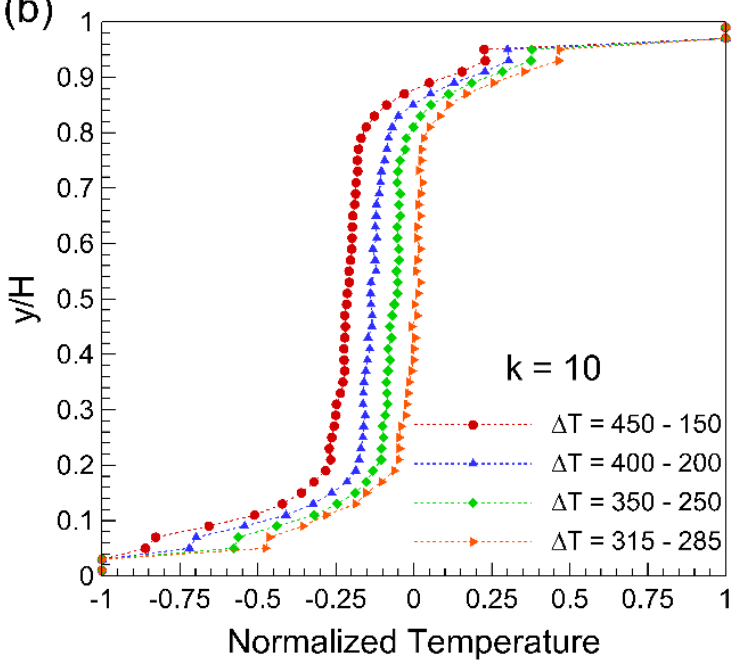

(d)

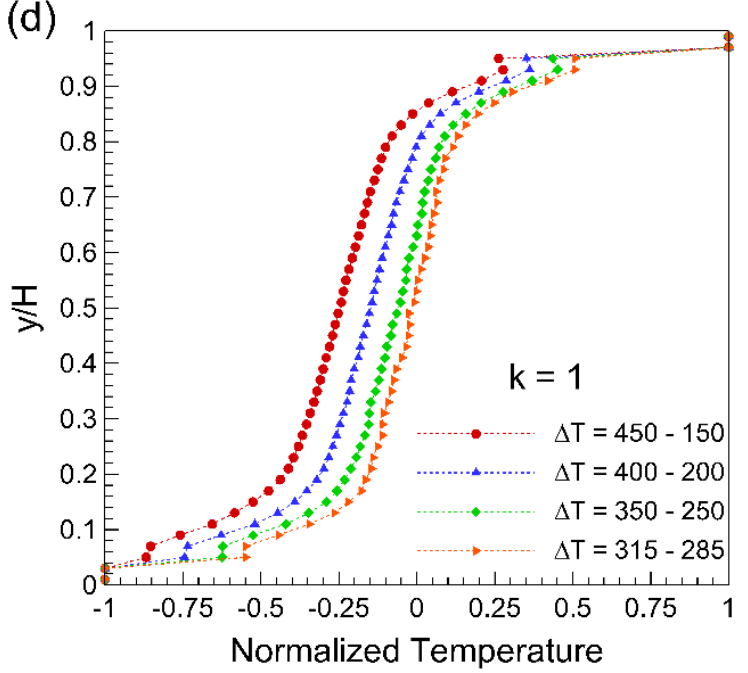

(f)

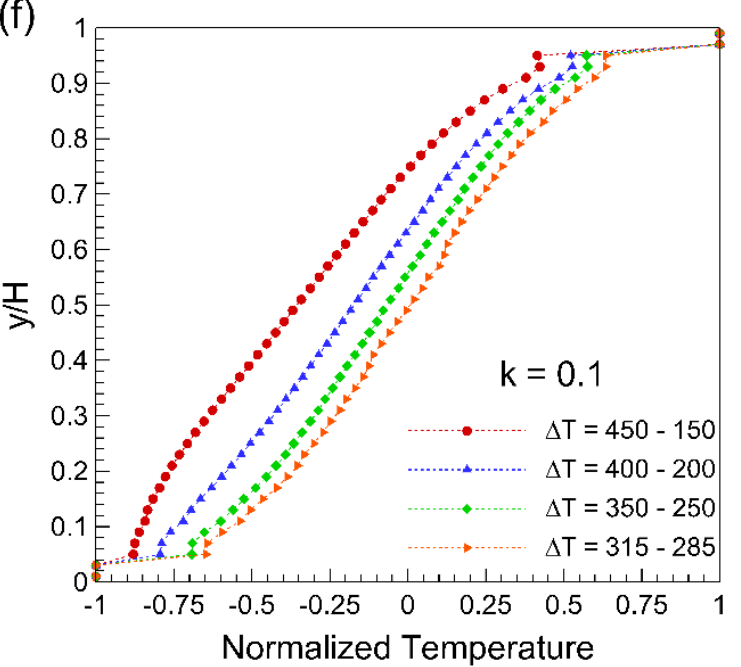

Fig. 4 Distribution of the gas density and temperature along the channel height for three modified Knudsen numbers (case A) 
The simulation parameters for $k=1$ are depicted in Table 2 . In the second set of simulation, the gas density increased in a way that the $k=1$ at the bulk region of the gas. As the Table 2 depicts, the required number of gas atoms for $k=1$ follows the same variation as for $k=10$. It is clear that for the case $\mathrm{A}$, the required number of gas atoms increases considerably in order to assure a constant gas density in the bulk region of the domain. Actually, for $\Delta T=30 \mathrm{~K}, 4800$ gas atoms are required for $k=1$, while for $\Delta T=300 \mathrm{~K}$, it increases to 5800 . According to Table 2, the bulk gas density is increased from 19.8 to $20.2 \mathrm{~kg} / \mathrm{m}^{3}$ as the wall temperature difference changes from $30 \mathrm{~K}$ to $300 \mathrm{~K}$ in case $\mathrm{B}$. This is the same behavior as observed for the case B and $k=10$. The normalized gas density and temperature distribution for case A are shown in Figs. 4c and $d$, respectively. A comparison between Figs. $4 \mathrm{a}$ and $4 \mathrm{c}$ shows that the distribution of the normalized gas density is unaffected by changing the density in the range of $k=1$ to $k=10$. Indeed, this interval corresponds to changing the gas density from 1.9 to $19 \mathrm{~kg} / \mathrm{m}^{3}$.

Finally, we increase the gas density until we attaint $k=0.1$ in the bulk region of the channel. As for case $\mathrm{A}$, the required number of gas molecules increases considerably from 48000 for $\Delta T=$ $30 \mathrm{~K}$ to 67000 for $\Delta T=300 \mathrm{~K}$ as it is shown in Table 2 . On the other hand, for case $\mathrm{B}$ the bulk gas density is increased from 200 to $203 \mathrm{~kg} / \mathrm{m}^{3}$ as the wall temperature difference increases from $30 \mathrm{~K}$ to $300 \mathrm{~K}$. Considering the bulk gas density for all cases in this set of simulations, it is observed that unlike in the previous set of simulations, we deal with a dense gas. Thus, we choose $2.7 \mathrm{~nm}$ as the cut off radius for the interaction between atoms [9].

Figures $4 \mathrm{e}$ and $\mathrm{f}$ depict the distribution of normalized gas density and temperature for various temperature differences of the case A along the height of the channel, respectively. A comparison between Figs. 4a, 4c and 4e shows that the distribution of the normalized gas density is a function of gas density, implemented temperature differences between the walls and even the wall temperature. Figures $4 \mathrm{a}$ and $4 \mathrm{c}$ depict that for the rarefied gas condition $(k=10$ and 1$)$, the normalized gas density starts to deviate from its bulk value by approximately $1 \mathrm{~nm}$ from each wall regardless of the implemented temperature differences. It is clear from Fig. 4e that by increasing the temperature differences between the walls and consequently decreasing the temperature of the lower wall, the dense gas near the lower wall behaves differently. Actually, for temperature differences of $30 \mathrm{~K}$ and $100 \mathrm{~K}$ the deviation of the normalized gas distribution occurs at about $1 \mathrm{~nm}$ from each wall.

As the temperature differences increase up to $200 \mathrm{~K}$ and consequently the lower wall temperature settles around $200 \mathrm{~K}$, the onset of density layering is observed. This causes the deviation of the gas normalized density from its bulk value at about $1.5 \mathrm{~nm}$ from the cold wall while for the hot wall, this is still about $1 \mathrm{~nm}$. For higher temperature differences, the lower wall temperature is decreased and this intensifies the density layering. Figure $4 \mathrm{e}$ clearly shows that for $\Delta T=300$ the overall behavior of the normalized gas distribution changes and the density distribution near the cold wall starts to deviate from its bulk value in about $2 \mathrm{~nm}$ from the cold wall. In this region, the gas density distribution experiences two peaks, where the first and more intense one appears at about $0.2 \mathrm{~nm}$ from the wall while the second one is at about $0.5 \mathrm{~nm}$ from the wall. On the other hand, it is also observed that near the hot wall, the maximum of the gas 
density occurs at $0.2 \mathrm{~nm}$ from each wall regardless of the implemented temperature difference between the walls.

The reason behind density layering phenomena is related to the interaction between the fluid and the cold wall atoms. The combination of the repulsive and the attractive forces between the particles leads to the various valleys and peaks which is a function of the fluid-wall interaction strength, structure of the wall atoms, the channel width and the fluid motion [45]. At the short distance from the wall atoms, the distance between the wall surface and the first peak of fluid atoms, strong repulsion occurs between the gas and the wall atoms. Therefore, the fluid atoms cannot get too close to the wall. Due to the attractive part of the Lennard-Jones potential between the cold wall and the fluid atoms, the fluid atoms forms the first peak at about $0.2 \mathrm{~nm}$ from the wall. At a short distance from the first peak, depletion of the fluid atoms is observed because of the strong repulsive force between the fluid atoms. Meanwhile, the attractive part of the LennardJones potential between the fluid atoms forms the second peak. It is obvious that the first peak is stronger than the second one. The reason refers to the fact that the first peak receives the attractive part of the Lennard-Jones potential of the wall-fluid interaction while the second peak receives the attractive part of the fluid-fluid interaction. Since the atomic structure of the wall is denser than the fluid, the resulting potential is much stronger which leads to such differences.

Figure $4 \mathrm{f}$ shows that as the gas density increases toward the continuum limit, the temperature moves toward a linear temperature profile. The deviation from linear variation for $\Delta T=300 \mathrm{~K}$ which is observed in this figure is due to the presence of density layering which is intense in this case near the lower wall.

Figures $5 \mathrm{a}$ and $\mathrm{b}$ depict the normalized gas density and temperature distribution for various temperature differences along the height of the channel for the case $\mathrm{B}$ and $k=10$. Since in this case the lower wall temperature is fixed at $300 \mathrm{~K}$ and the upper wall temperature is increased, the maximum gas density near the lower wall is about 3.18 times the bulk gas density for all temperature differences. For the upper wall, the maximum density varies from 2.83 times of the bulk gas density for $\Delta T=30 \mathrm{~K}$ to 1.71 for $\Delta T=300 \mathrm{~K}$. This is due to the fact that by increasing the temperature difference between the walls, the upper wall temperature increases and the residence time of the gas molecules in the wall force field region decreases considerably. This results in the reduced gas density there. Direct consequences of this density reduction near the upper wall can be observed in Table 2, where the bulk gas density increases from 1.95 to $2.1 \mathrm{~kg} / \mathrm{m}^{3}$ as the temperature difference changes from $30 \mathrm{~K}$ to $300 \mathrm{~K}$. The normalized temperature distribution conveys the gas density distribution as it is shown in Fig. 5b. Since the normalized gas density reduces gradually as the temperature increases near the upper wall, a reduction in the maximum of the gas normalized temperature near the upper wall is observed. The main reason refers to the fact that by reducing the maximum density near the upper wall, the residence time of gas molecules in the wall force field region decreases and the collision rate between the gas and the wall decreases considerably. Consequently, the gas atoms are not able to reach the wall temperature so the difference between the maximum gas temperature and the wall is increased. 
The distribution of normalized density and temperature for various temperature differences for the case B and $k=1$ are shown in Figs. 5c and d, respectively. Comparison with Fig. 5a proves the previously observed phenomena which imply that the distribution of the normalized gas density is approximately unaffected by changing the gas density in the range of $k=1$ to $k=10$. In addition, Fig. $5 \mathrm{~d}$ shows that the maximum of the gas temperature near the top and bottom walls is getting closer to its corresponding wall temperature. This is in contrast to Fig. $5 \mathrm{~b}$.

The normalized gas density and temperature distribution for various temperature differences along the height of the channel are shown in Figs. 5e and f, respectively, for the case B. It should be noted that since the temperature of the lower wall is constant $(300 \mathrm{~K})$, a comparison between the maximum gas densities near the lower wall in Figs. 5a, 5c and 5e reveal the relative importance of the interaction between the wall force field and the wall molecules. It is obvious that as we move from $k=10$ to $k=0.1$, the maximum of the normalized gas density changes from 3.18 to 2.68. This reduction in the maximum normalized density is because in the rarefied gas case, the gas atoms are highly affected by the wall force field. As in the data shown in the Fig. 2a, there are not many gas atoms in the neighboring area to interact with it, and the motion of the gas atoms is highly affected by the wall force field leading to a maximum normalized density of about 3.18. As the gas becomes denser (similar to the Fig. 2c), the number of neighboring atoms for each individual gas atom is increased so the net force field is a combination of the wall force field and the forces due to the neighboring gas atoms. This leads to a reduction in the maximum gas density distribution to the value of 2.68. This demonstrates that increasing the gas density reduces the wall force field effect on the gas medium.

The effect of increasing density on the distribution of the temperature profile at a constant wall temperature $(300 \mathrm{~K})$ is also observed in the comparison between the Figs. $5 \mathrm{~b}, 5 \mathrm{~d}$ and $5 \mathrm{f}$. It is clear that as the gas density is increased in the domain by moving from $k=10$ to $k=0.1$, the differences between the maximum gas temperature near the wall and the wall temperature ( $300 \mathrm{~K})$ in its normalized form decreases from 0.5 to 0.35, i.e.it gets closer to the wall temperature. Actually, as the gas density is increased in the domain by moving from $k=10$ to $k=0.1$, the gas-wall collisions increase drastically which leads to the enhancement of heat transfer through the gas medium. The increased heat transfer leads to a reduction in the difference between the maximum temperature in the gas and the wall temperature for all temperature differences between the walls.

An interesting phenomenon is observed as the heat transfer mechanism in the gas is considered. As it is shown in the Fig. $4 \mathrm{~b}$ for the rarefied gas case $(k=10)$, two distinct regions are observed in the distribution of the gas temperature along the channel height. It is clear that in the wall force field region near each wall, a gradient in the temperature distribution is observed and the temperature changes with a remarkable slope. In contrast, sufficiently far away from the channel walls, a uniform temperature is observed in the bulk region. Indeed, due to the increase in residence time of the gas atoms in the wall force field region, the accumulation of the gas atoms occurs as it is shown in the Fig. 4a. Thus, collisions between the gas atoms increase notably and with it the diffusive heat transfer. In contrast, the uniform temperature distribution in the bulk region of the 
gas is due to the fact that the gas is rarefied and collisions are less frequent, so that heat transfer is dominated by direct atom-wall collisions corresponding to the ballistic heat regime. As the gas becomes denser $(k=0.1)$ as shown in Fig. $4 \mathrm{f}$, the intermolecular gas-gas collisions are increased even in the bulk region and the heat transfer mechanism is changed to diffusion-like along the channel height. The direct consequence of this change in heat transfer mechanism can be observed in the temperature gradient in the bulk region of the gas which shows the gas-gas collision nature. It should be noted that a similar behavior is also observed by comparing Figs. 5b, 5d and $5 f$.

Another interesting observation is about the required number of $n_{s s c}$ and $n_{\text {avr }}$ for each case. Based on Table 2, for $k=10$ and the temperature differences in the order of $30 \mathrm{~K}$, the required number for $n_{s s c}$ and $n_{\text {avr }}$ are about $10 \times 10^{6}$ and $50 \times 10^{6}$, respectively. As the temperature difference between the walls is increased, $n_{s s c}$ and $n_{\text {avr }}$ are decreased to $2 \times 10^{6}$ and $5 \times 10^{6}$, respectively and higher number of averaging does not change the averaged quantity. This is due to the fact that as the temperature difference between the walls is increased, the resulting heat flux through the gas is enhanced notably and a change in the gas kinetic energy occurs more rapidly in comparison with lower temperature differences between the walls. The higher changes in kinetic energy mean the higher changes in the gas atoms velocity and temperature so the averaged quantities converge more rapidly to its final value with a lower number of required averaging steps.

Considering $k=0.1$ and temperature differences in the order of $30 \mathrm{~K}$ (see Table 2), it is shown that the required number for $n_{s s c}$ and $n_{\text {avr }}$ are reduced considerably to $1 \times 10^{6}$ and $2 \times 10^{6}$, respectively, in comparison with the same temperature differences when $k=10$. The reason of such behavior is the fact that as the gas density is increased, the number of the gas atoms increases and gas-wall collisions become more frequent which results in a higher heat transfer rate through the gas medium. Simultaneously, the gas-gas collision rate increases due to the higher density. The combination of these two phenomena leads to the reduced required number for the $n_{s s c}$ and $n_{\text {avr }}$ in this case. In contrast, it should be noted that while the required number of time steps for the averaging is reduced considerably, due to the increment in the number of the gas atoms in the domain from 480 to 48000 , each time step takes substantially more computational time.

It should be noted that the Knudsen number is determined in our simulations based on a trial and error method. Considering the Figures 4 and 5, it is clearly shown that the gas density distribution is non-uniform along the channel height due to the presence of the wall force field which effectively results in a position dependent Knudsen number. To the best of the knowledge of the authors, there is no analytical formula or method to determine the exact number of the gas atoms for a desired Knudsen number under such circumstances and a systematic parameter study is the only available method within the literature $[10,11,13,14,37]$. In this method, the number of gas atoms is varied until the desired gas density (which corresponds to the desired Knudsen number) is achieved in the center of the channel. It should be noted that small differences between the calculated modified Knudsen number and $k=0.1,1$ and 10 are due to the trial and error nature of the Knudsen number determination. Several simulations are conducted to find the gas atom number in each case and as Table 2 depicts, the overall difference with the desired modified Knudsen number is below $10 \%$ for all cases. The authors believe that the exact number of atoms 
in each case is an important output of our research since these numbers are found through several time-consuming simulations for each case and can be used by other researchers to produce the desired Knudsen number. The following discussion clearly shows the relation between the gas density and the desired Knudsen number. In rarefied gas dynamics, the mean free path of the gas medium is defined as [1]

$$
\lambda=\frac{1}{\sqrt{2} \pi d^{2} n},
$$

where $d$ is the diameter of the gas atoms and $n$ denotes the number density. Considering the definition of the modified Knudsen number, $k=(\sqrt{\pi} / 2) K n$, we have

$$
k=\frac{1}{\sqrt{8 \pi} d^{2} n l},
$$

where $l$ is the characteristic length scale of the domain which is considered as the channel height of $5.4 \mathrm{~nm}$ in our simulations for all cases. Moreover, if $N$ is considered as the total number of the gas atoms, the number density is

$$
\begin{aligned}
& \rho=\frac{\mathrm{m}}{V}=\frac{\mathrm{N} \times m_{\text {atom }}}{V}, \\
& n=\frac{\mathrm{N}}{V}=\frac{\rho}{m_{\text {atom }}} .
\end{aligned}
$$

Considering the fact that $d=0.3405 \mathrm{~nm}$ and $m_{\text {atom }}=6.63 \times 10^{-26} \mathrm{Kg}$ for argon gas atoms and in all simulations throughout the manuscript, the final formula for the modified Knudsen number becomes

$$
k=\frac{m_{\text {atom }}}{2 \sqrt{2 \pi} d^{2} \rho l}=\frac{6.63 \times 10^{-26}}{\sqrt{8 \pi} \times\left(0.3405 \times 10^{-9}\right)^{2} \times \rho \times\left(5.4 \times 10^{-9}\right)}=\frac{21.11}{\rho},
$$

Equation (12) is used in all simulations through the manuscript to define the modified Knudsen number based on the gas density in the center of the channel. Table 2 summarizes the effect of the gas temperature on the required number of argon gas atoms leading to the desired gas density in the center of the channel.

Figure 6 displays the variation of the induced heat flux through the gas and the corresponding effective thermal conductivity of the gas medium as a function of temperature difference between the walls for all three Knudsen numbers. Since the Knudsen number is relatively high, the concept of "effective thermal conductivity" is introduced as $K_{e f f}=J_{y} \Delta T / H$ [18]. It is clear that for all Knudsen numbers, case B shows higher values of the heat flux and the effective thermal conductivity in comparison with the case A that refers to the differences in the distribution of the gas density along the channel height between the case A and B. As the temperature difference between the walls changes from $30 \mathrm{~K}$ to $300 \mathrm{~K}$, the difference between the induced heat fluxes through the gas increase between the two cases. As an example, the heat flux through the rarefied gas for the case A of $k=10$ at $\Delta T=300$ is $10.3 \mathrm{MW} / \mathrm{m}^{2}$ while for the case $\mathrm{B}$, it increases by about $24 \%$ to $12.8 \mathrm{MW} / \mathrm{m}^{2}$. 
(a)

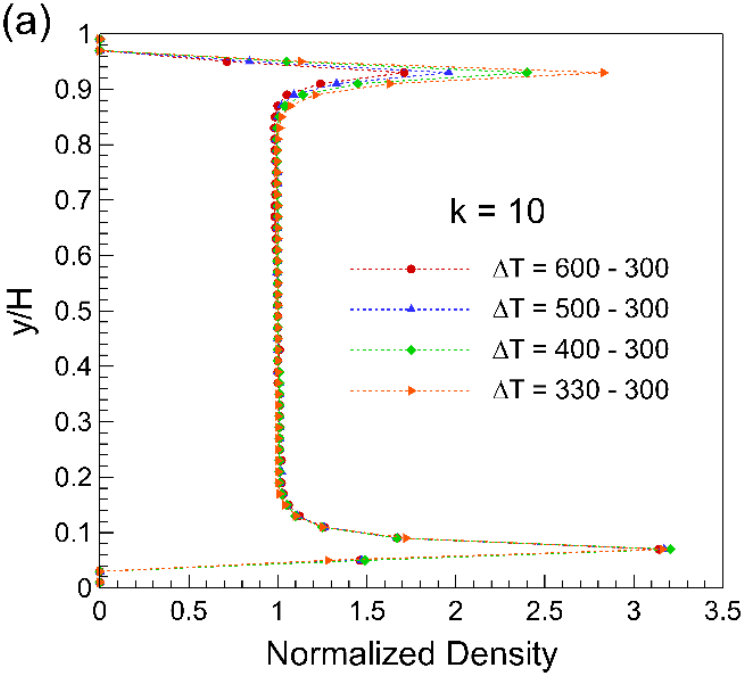

(c)

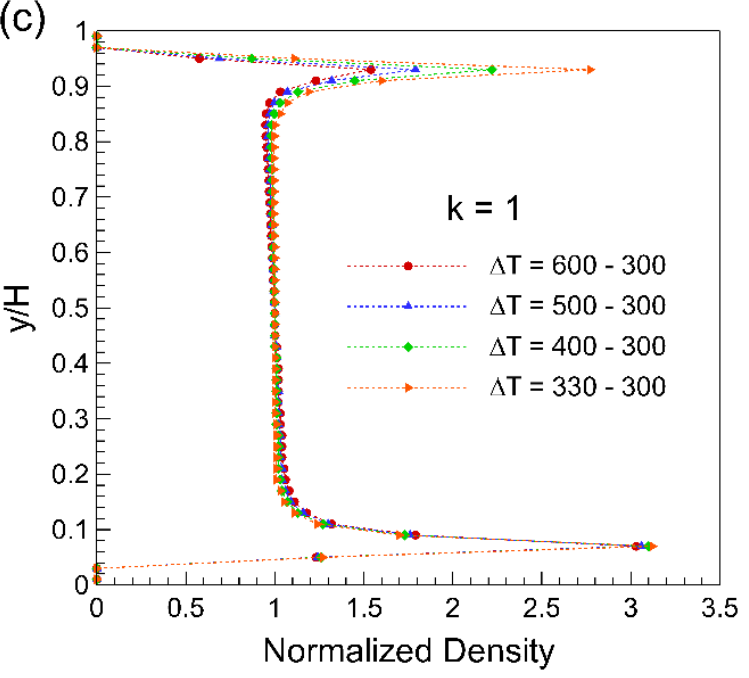

(e)

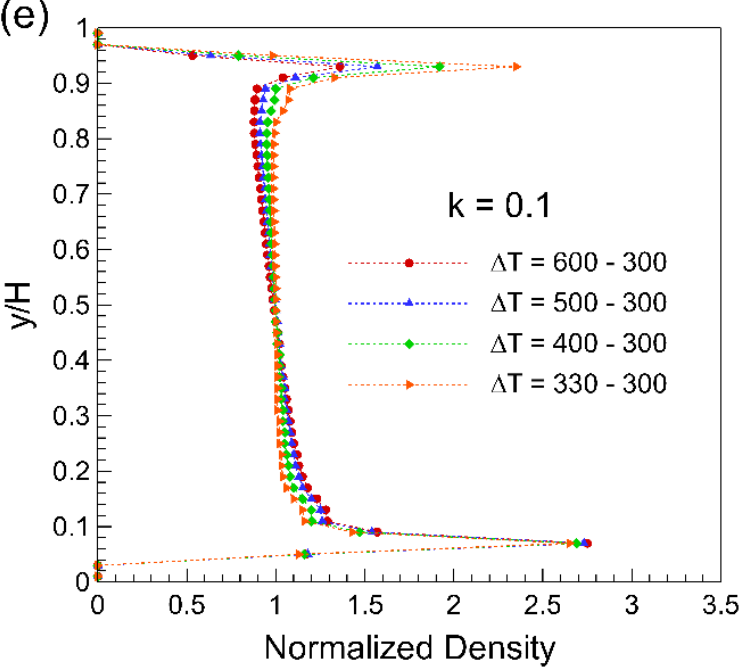

(b)

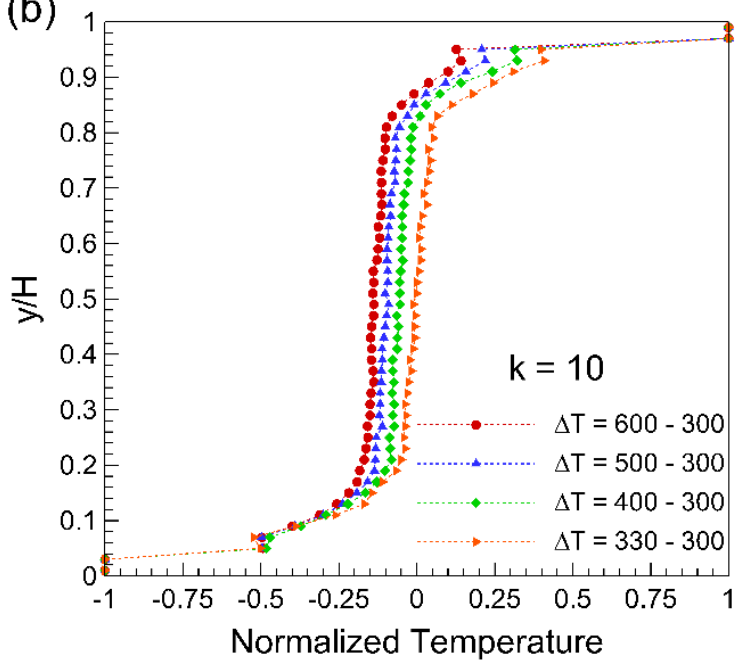

(d)

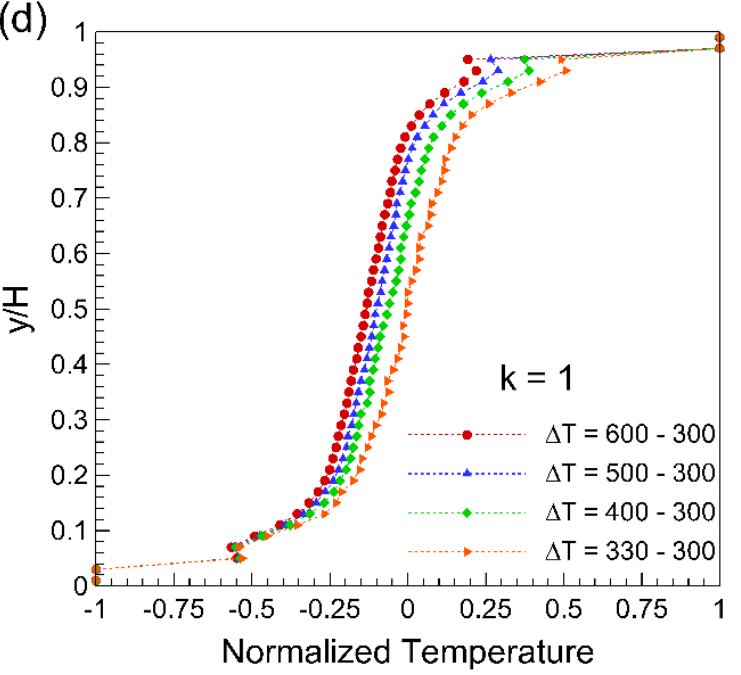

(f)

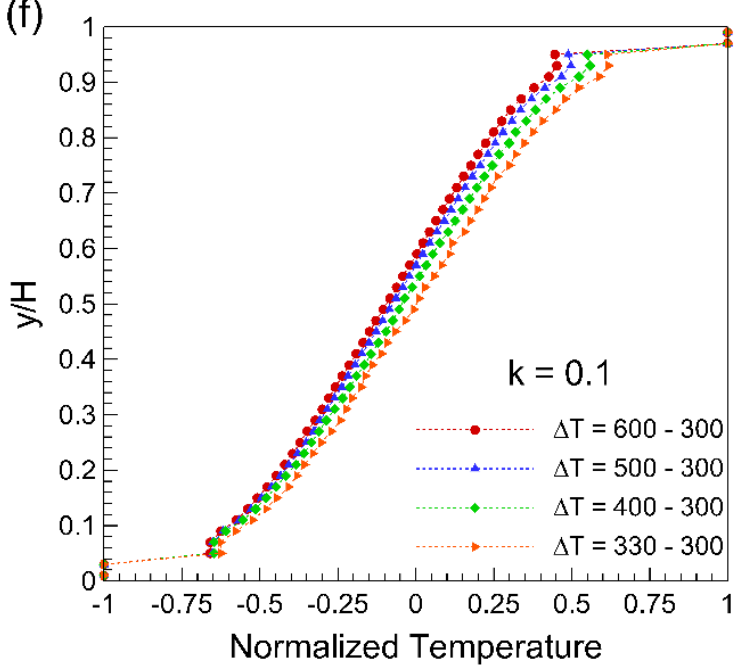

Fig. 5 Distribution of the gas density and temperature along the channel height for three modified Knudsen numbers (case B) 
For the case $\mathrm{A}$ of $k=0.1$ at $\Delta \mathrm{T}=300$ where the gas is dense, the induced heat flux through the gas is $676 \mathrm{MW} / \mathrm{m}^{2}$ and increases by about $23 \%$ to $834 \mathrm{MW} / \mathrm{m}^{2}$ for the case B. Furthermore, Fig. 6 reveals that the overall trend of the heat flux curve against wall temperature differences is the same and it is simply shifted up as the gas becomes denser. It is clear that as the gas becomes denser, the corresponding effective thermal conductivity is also increased. Actually, for $k=10,1$ and $0.1, K_{\text {eff }}$ is approximately $0.18,1.85$ and $12 \mathrm{~mW} / \mathrm{mK}$ for $\Delta T=30$. The effect of changing the temperature differences is also observed in this part. For the case $\mathrm{B}$ of $k=10$ and 0.1 , by increasing the temperature difference to $300 \mathrm{~K}, K_{\text {eff }}$ become approximately 0.22 and $14.5 \mathrm{~mW} / \mathrm{mK}$, respectively, which corresponds to $22 \%$ or $21 \%$ deviation in comparison with $\Delta T=30$.

(a)

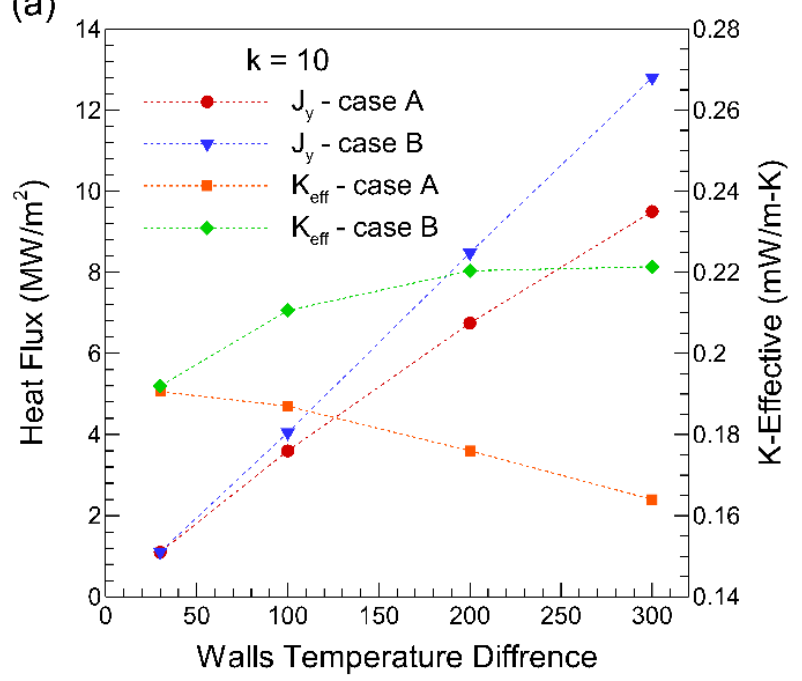

(b)

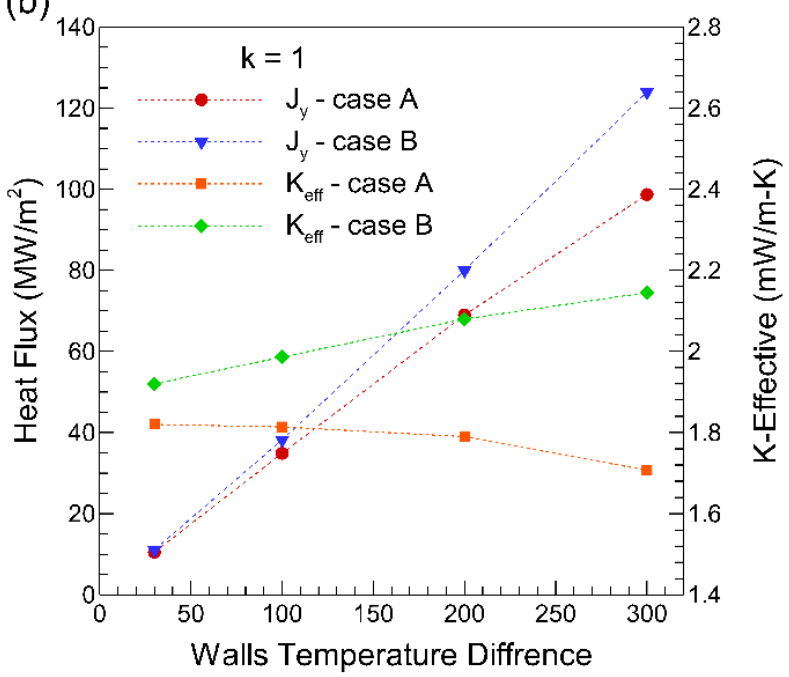

(c)

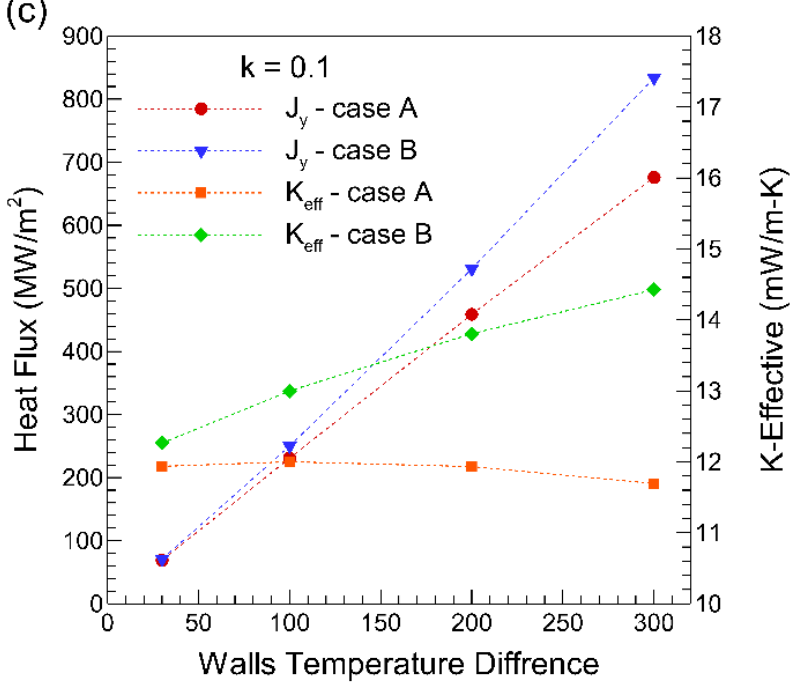

Fig. 6 Heat flux and effective thermal conductivity against temperature differences between the walls for $k=10$ (a), $k=1$ (b) and $k=0.1$ (c) 
The same trend is observed for $k=1$ which implies that in nanoscale-confined gas heat transfer, the calculated effective thermal conductivity is a function of wall temperature differences. The reason refers to the fact that as the walls temperature difference increases, the distribution of the gas atoms along the channel height varies which leads to differences in the determination of the effective thermal conductivity. Due to the same reason, for the case A and all three Knudsen numbers, a small reduction occurs in the calculated effective thermal conductivity as the temperature difference between the walls increases from 30 to $300 \mathrm{~K}$.

In order to investigate the heat transfer mechanism along the channel height more precisely, the distribution of the thermal conductivity along the channel height is considered by dividing the channel height into three parts based on the wall force field penetration depth. Based on the potential which is used to simulate the interaction between wall atoms and gas, the wall force field penetrates into the gas in the order of $1 \mathrm{~nm}$ [8-10]. According to this, the normalized temperature profile can be divided into three main regions as it is shown in Figs. 7a and b. Actually, the regions A-B and C-D are the regions that the wall force field penetrate into the gas medium while B-C denotes the bulk region. This implies that if the effective thermal conductivity formula is applied to each part separately, the ELTC of each region is specified more precisely.

It should be considered that according to the Figs. 7a and b, the ELTC between the bottom wall and line $\mathrm{A}$ in addition to the top wall and line $\mathrm{D}$ is not calculated due to an insufficient number of gas atoms there as it is observed in the normalized density profile for all cases. It should be considered that the temperature profiles in Figs. 7a and $\mathrm{b}$ are depicted as a sample and the abovementioned procedure is applied to all normalized temperature profiles throughout the paper. The resulting ELTC for each case in the middle of its corresponding region is shown in Figure 8.

(a)

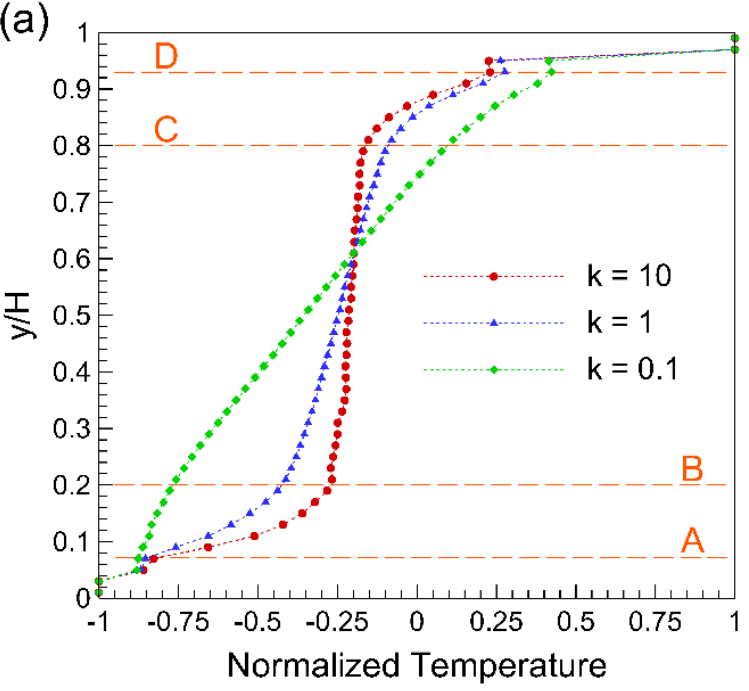

(b)

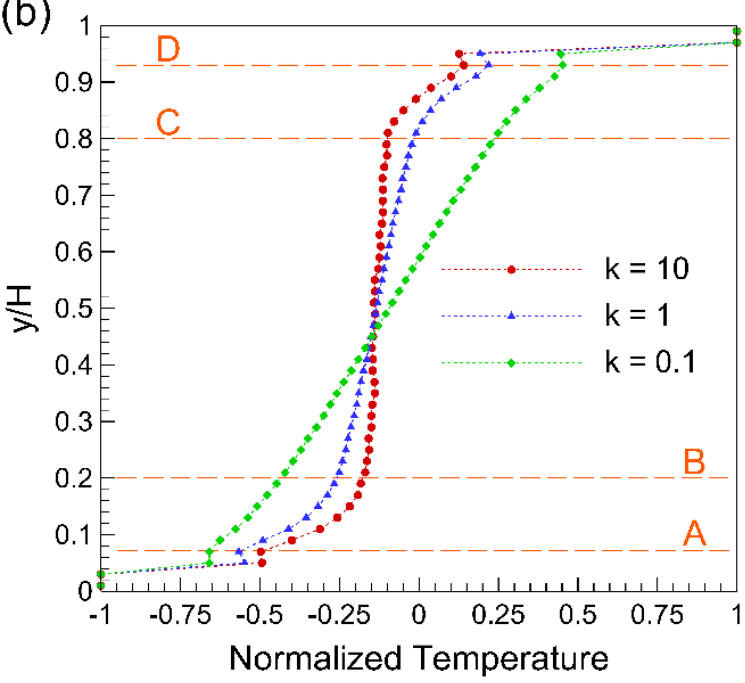

Fig. 7 Schematic division of the channel height based on the wall force field penetration depth for temperature differences of $300 \mathrm{~K}$ between the channel wall in the case A (a) and the case B (b) 
(a)

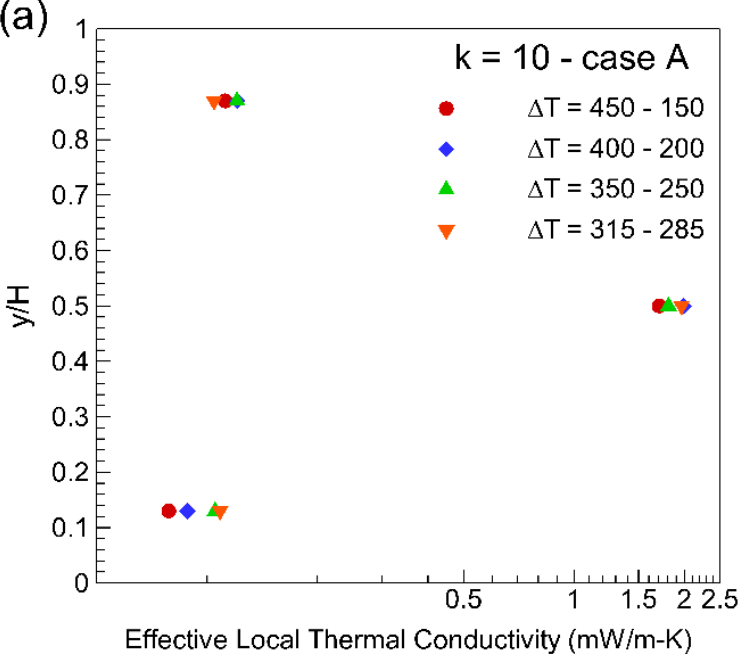

(c)

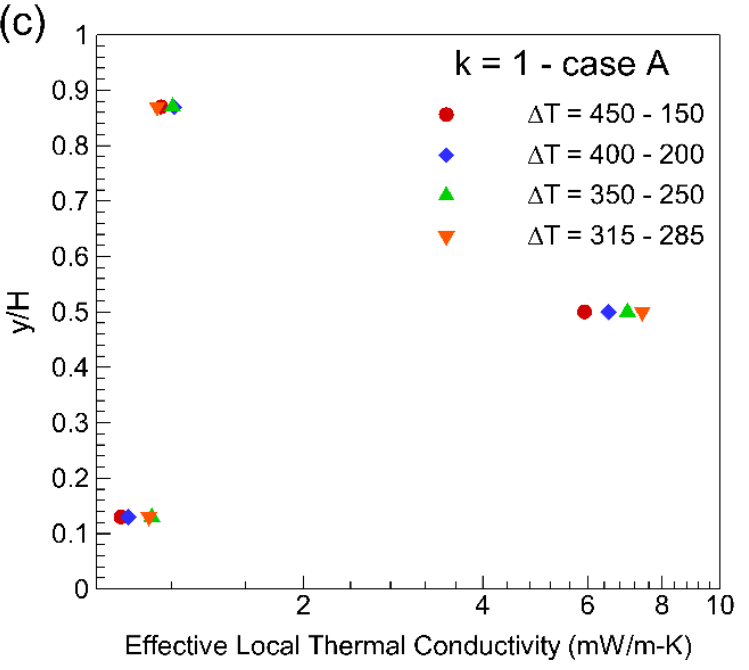

(e)

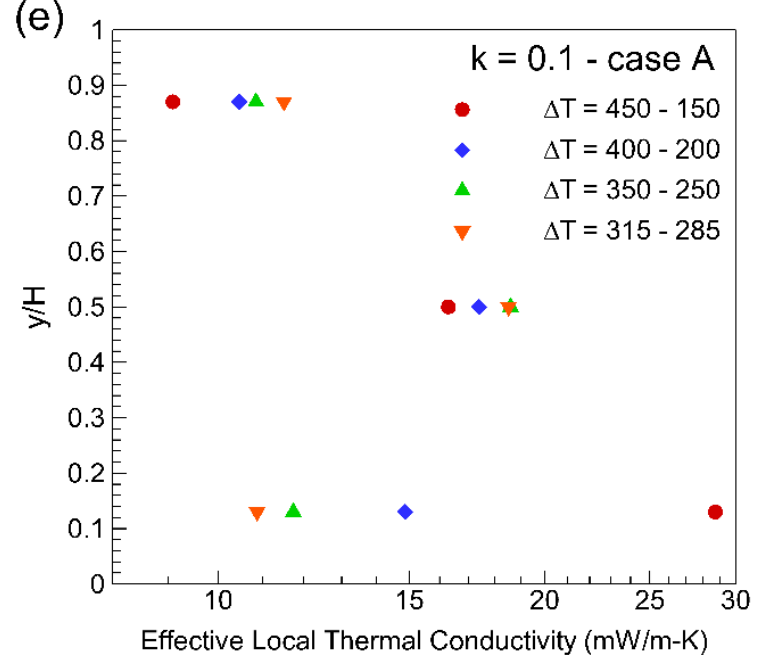

(b)

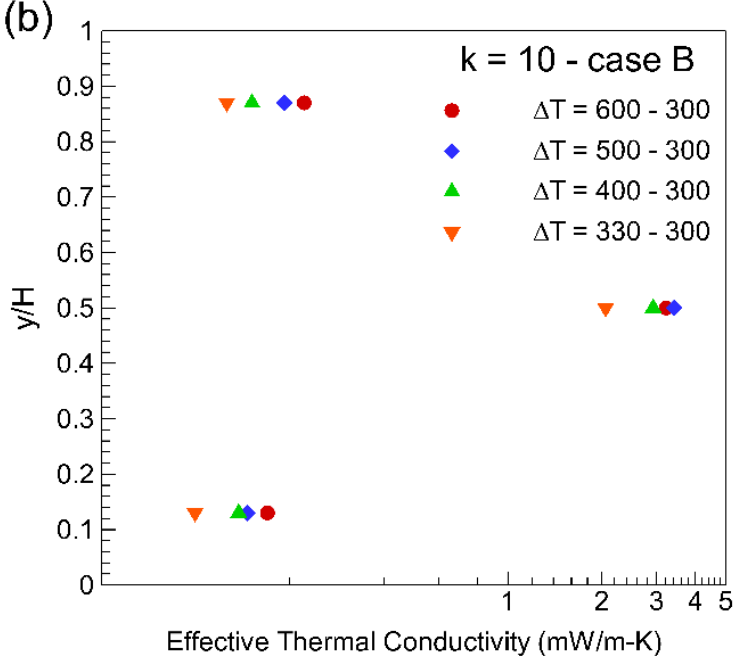

(d)

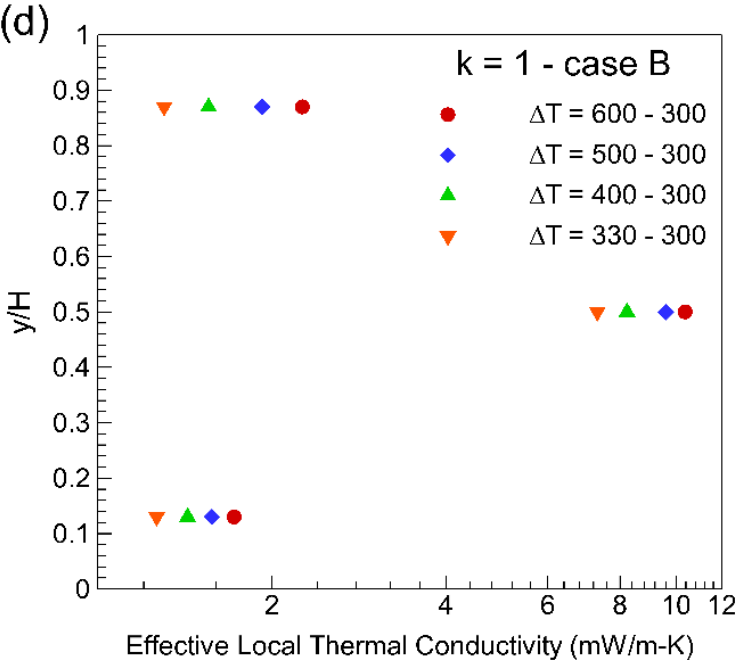

(f)

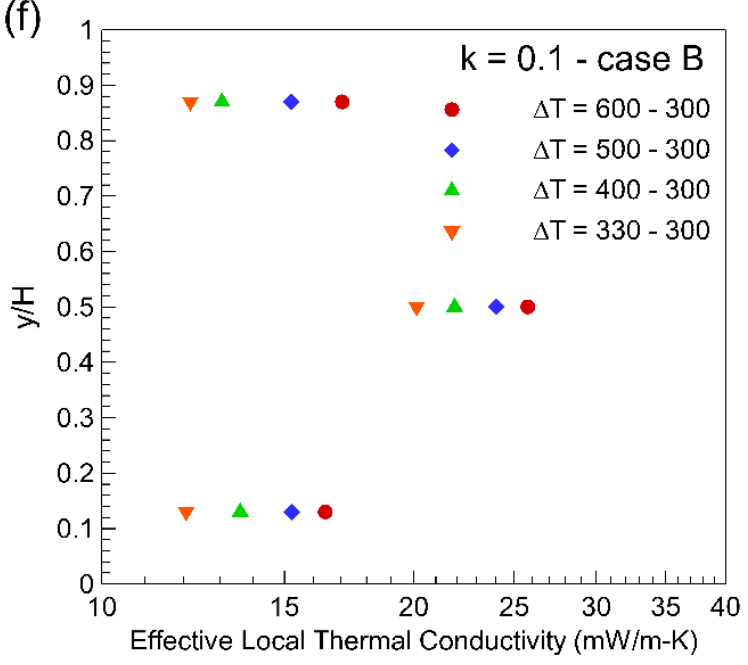

Fig. 8 Distribution of the effective local thermal conductivity of the gas along the channel height for three modified Knudsen numbers 
Figures $8 \mathrm{a}$ and $\mathrm{b}$ clearly depict the existence of the two different transport mechanisms along the channel height as discussed before. For both cases, it is obvious that the ELTC of the gas in the wall force field region changes in the range of 0.08 to $0.22 \mathrm{~mW} / \mathrm{mK}$ while in the bulk region, it changes in the range of 1.7 to $3.4 \mathrm{~mW} / \mathrm{mK}$ which is approximately one order of magnitude more. The general trend is in complete accordance with the variation of local thermal conductivity along the channel height in nanoconfined liquid medium [19].

In our study, this difference are due to the fact that as previously mentioned, the ballistic transport mechanism is dominant in the bulk region and the gas atoms transfer heat between the hot and cold medium without any collision to other gas atoms so that the ELTC increases. Besides, in the wall force field region the gas density is higher due to an increase in the residence time of the gas atoms. This leads to an increase in the number of gas-gas and gas-wall collisions which shows the existence of the diffusive transport mechanism. A direct effect of increased residence time is a considerable reduction in the ELTC of the gas in this region. Similar trend is also observed for $k=1$ when the Figs. $8 \mathrm{c}$ and $\mathrm{d}$ are considered. The increment in the magnitude of the ELTC that is observed in comparison with $k=10$ is due to the increase in the number of gas atoms. In the wall force field region, the ELTC changes in the range of 0.1 to $2.25 \mathrm{~mW} / \mathrm{mK}$ while in the bulk region, it ranges from 5.92 to 10.37 . This shows the presence of the diffusive and ballistic heat transport mechanism in the wall force field and bulk region, respectively.

As the gas becomes denser and $k=0.1$ is reached, it is observed from Figs. 8e and $\mathrm{f}$ that the ELTC near each wall varies in the same order of magnitude as the bulk region which is the sign of a uniform transport mechanism along the channel height. Indeed, the diffusion mechanism governs the gas and the effect of the wall force field on the gas atoms is reduced significantly due to the increment of the forces due to the other gas atoms. It is interesting to note that for $k=0.1$ and $\Delta T=450-150$, the ELTC near the cold wall is higher than its corresponding value in the bulk region. This is due to the onset of the density-layering phenomenon which can be observed in Fig. 4e. Under this condition, the dense gas near the cold wall behaves like a liquid, i.e. the ELTC in that region shows a notable increase. Furthermore, considering Figs. 8b, $d$ and e shows that as the upper wall temperature is increased, the ELTC along the channel height is increased. The reason refers to the fact that as the upper wall temperature changes from 300 to $600 \mathrm{~K}$, the induced heat flux through the gas is increased which leads to more energy for each gas atom and thus a higher velocity for the atoms which results in better transport characteristics of the gas.

Using the experimental thermal conductivity of argon gas $\left(K_{C}\right)$ [46] at the desired density and mean temperature of walls as the characteristic temperature of the gas medium from Tables 2 , the effective thermal conductivities are normalized according to Fig. 9. This figure validates our result against the experimental data by showing the increment in the value of normalized effective thermal conductivity as it moves toward continuum limit. As the modified Knudsen number changes from 10 to 0.1 , the normalized effective thermal conductivity changes approximately from 0.01 to 0.5 which shows the effect of the wall force field is more pronounced in the rarefied gas condition in comparison with the dense gas case. The same trend is also observed in a comparison between the ELTC of the corresponding Knudsen number in Fig. 8. 
Actually, for the $k=10$, the ELTC in the wall force field regions are one order of magnitude smaller than the bulk value. This significant reduction of the ELTC in the wall force field region of the rarefied gas case in comparison with corresponding bulk value leads to a drastic reduction (approximately 1/100 times) of the overall effective thermal conductivity of the nanoconfined gas medium in comparison with the experimental data of the argon gas at the same density and characteristic temperature. On the other hand for the $k=0.1$, while the ELTC in the wall force field regions is still smaller than corresponding bulk value, they are in the same order of magnitude. Therefore, the reduction in the overall effective thermal conductivity of the nanoconfined dense gas medium in comparison with the experimental data of the argon gas at the same density and characteristic temperature is limited (approximately $1 / 2$ times).

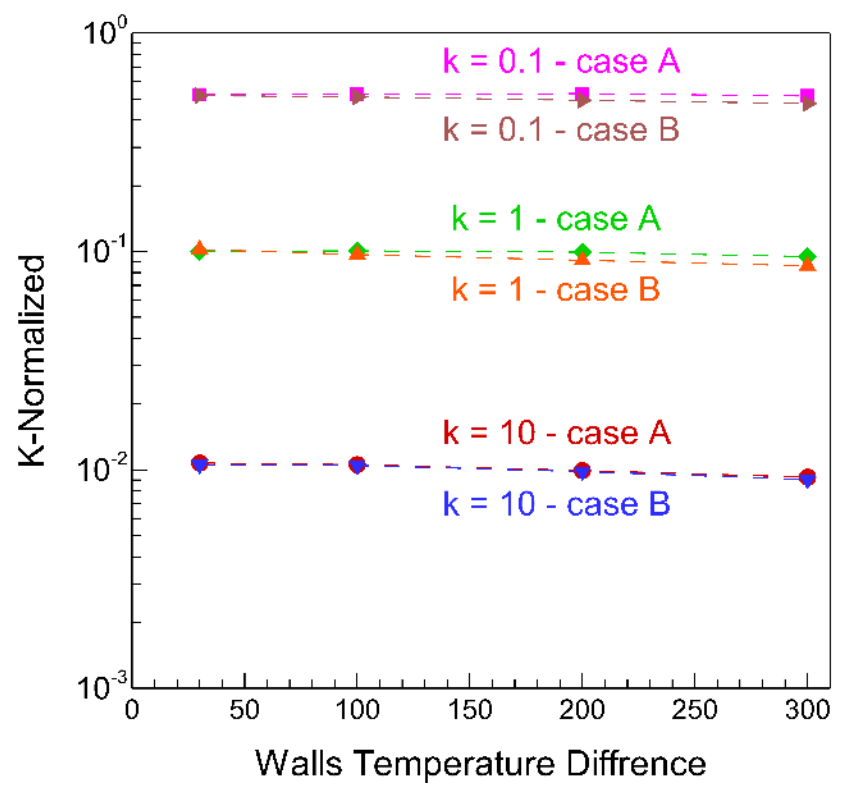

Fig. 9 Normalized effective thermal conductivity against temperature differences between the walls for $k=10,1$ and 0.1

\section{Conclusion}

The static argon gas confined in a nanochannel is subjected to various temperature differences between the channel walls to investigate the simultaneous effect of the wall force field and the heat transfer on the distribution of different gas properties such as density, temperature, heat flux and effective thermal conductivity by the use of the molecular dynamics simulations. The channel height is $5.4 \mathrm{~nm}$ and the gas density varies in such a way that the modified Knudsen number becomes 10,1 and 0.1 . It is observed that since approximately $40 \%$ of the channel height is affected by the wall force field, the distribution of the gas atoms along the channel height and heat transfer characteristic of the gas medium strongly depends on an interplay between the wall force field, the wall temperature and the heat flux across the gas region. Considering the increase in the residence time of the gas atoms near each wall due to the wall force field, it is shown that this would be changed drastically by a variation of the adjacent wall temperature which leads to an 
unequal accumulation and scattering of gas atoms near the cold and hot wall, respectively. This difference causes the normalized temperature profile to be dependent on the temperature difference between the walls. The behavior of the normalized gas density and temperature profiles near each wall reveals that as the gas density is increased, the effect of the wall force field on gas atoms reduces significantly since the gas force field becomes stronger. A change in the heat transfer mechanism is another phenomenon which is observed as the gas density increased. While ballistic heat transport leads to a uniform temperature profile in the bulk region of the rarefied gas $(k=10)$, it is observed that diffusive heat transport in the wall force field region leads to a linear variation of the temperature profile. Furthermore, it is shown that the ELTC in the bulk region is about one order of magnitude higher than the corresponding value in the wall force field region which is another sign of the dual heat transport mechanism in the rarefied gas. In contrast, as the gas becomes dense $(k=0.1)$, the temperature profile shows a linear variation along the channel height which is a characteristic of diffusion dominated heat transfer. It should be considered that for determination of the thermal conductivity at a desired temperature of the gas, only small temperature differences $(\sim 30 \mathrm{~K})$ could be applied on the walls since the effective thermal conductivity and ELTC are strongly dependent on the temperature.

\section{Conflict of interest}

I hereby declare on behalf of all the co-authors of this paper and myself that no conflict of interests with anything or anyone exists for what relates to the paper itself or to the research that made it possible.

\section{References}

[1] G. Karniadakis, A. Beskok, A. Narayan, Microflows and Nanoflows, Springer, 2005. doi:10.1007/0-387-28676-4.

[2] M. Rebay, Y. Kabar, S. Kakaç, Microscale and Nanoscale Heat Transfer: Analysis, Design, and Application, CRC Press, 2016. doi:doi:10.1201/b19261-12.

[3] Z.M. Zhang, Nano/microscale Heat Transfer, McGraw-Hill, 2007.

[4] S. Fukui, R. Kaneko, Analysis of ultra-thin gas film lubrication based on linearized Boltzmann equation: first report - derivation of a generalized lubrication equation including thermal creep flow, J. Tribol. 110 (1988) 253-261. doi:10.1299/kikaic.53.829.

[5] M. Cieplak, J. Koplik, J.R. Banavar, Applications of statistical mechanics in subcontinuum fluid dynamics, Phys. A Stat. Mech. Its Appl. 274 (1999) 281-293. doi:10.1016/S0378-4371(99)00308-8.

[6] M. Cieplak, J. Koplik, J.R. Bavanar, Molecular dynamics of ows in the Knudsen regime, Physica A. 287 (2000) 153-160.

[7] M. Cieplak, J. Koplik, J.R. Banavar, Boundary Conditions at a Fluid-Solid Interface, Phys. Rev. Lett. 86 (2001) 803-806. doi:10.1103/PhysRevLett.86.803.

[8] M. Barisik, B. Kim, A. Beskok, Smart wall model for molecular dynamics simulations of nanoscale gas flows, Commun. Comput. Phys. 7 (2010) 977-993. doi:10.4208/cicp.2009.09.118. 
[9] M. Barisik, A. Beskok, Equilibrium molecular dynamics studies on nanoscale-confined fluids, Microfluid. Nanofluidics. 11 (2011) 269-282. doi:10.1007/s10404-011-0794-5.

[10] M. Barisik, A. Beskok, Molecular dynamics simulations of shear-driven gas flows in nano-channels, Microfluid. Nanofluidics. 11 (2011) 611-622. doi:10.1007/s10404-0110827-0.

[11] M. Barisik, A. Beskok, Scale effects in gas nano flows, Phys. Fluids. 26 (2014) 052003. doi:10.1063/1.4874678.

[12] M. Barisik, A. Beskok, Molecular free paths in nanoscale gas flows, Microfluid. Nanofluidics. 18 (2015) 1365-1371. doi:10.1007/s10404-014-1535-3.

[13] M. Barisik, A. Beskok, "Law of the nano-wall" in nano-channel gas flows, Microfluid. Nanofluidics. 20 (2016) 1-9. doi:10.1007/s10404-016-1713-6.

[14] M. Barisik, A. Beskok, Surface-gas interaction effects on nanoscale gas flows, Microfluid. Nanofluidics. 13 (2012) 789-798. doi:10.1007/s10404-012-1000-0.

[15] W.D. Zhou, B. Liu, S.K. Yu, W. Hua, Rarefied-gas heat transfer in micro- and nanoscale Couette flows, Phys. Rev. E - Stat. Nonlinear, Soft Matter Phys. 81 (2010) 1-7. doi:10.1103/PhysRevE.81.011204.

[16] H. Li, N. Sagawa, A novel active-head slider with a shear-mode PZT actuator and dual thermal actuator, IEEE Trans. Magn. 49 (2013) 3771-3774. doi:10.1109/TMAG.2013.2241400.

[17] H. Li, S. Shen, An efficient thermal actuator design for the thermal flying height control slider, Microsyst. Technol. 20 (2014) 1647-1651. doi:10.1007/s00542-014-2205-9.

[18] J.-P.M. Peraud, C.D. Landon, N.G. Hadjiconstantinou, Monte Carlo Methods for solving the Boltzmann transport equation, Annu. Rev. Heat Transf. (2014) 205-265. doi:10.1615/AnnualRevHeatTransfer.2014007381.

[19] F. Sofos, T. Karakasidis, A. Liakopoulos, Transport properties of liquid argon in krypton nanochannels: Anisotropy and non-homogeneity introduced by the solid walls, Int. J. Heat Mass Transf. 52 (2009) 735-743. doi:10.1016/j.ijheatmasstransfer.2008.07.022.

[20] X. Huang, X. Huai, S. Liang, X. Wang, Thermal transport in Si/Ge nanocomposites, J. Phys. D. Appl. Phys. 42 (2009) 095416. doi:10.1088/0022-3727/42/9/095416.

[21] A.R. bin Saleman, H.K. Chilukoti, G. Kikugawa, M. Shibahara, T. Ohara, A molecular dynamics study on the thermal energy transfer and momentum transfer at the solid-liquid interfaces between gold and sheared liquid alkanes, Int. J. Therm. Sci. 120 (2017) 273288. doi:10.1016/j.ijthermalsci.2017.06.014.

[22] F. Faraji, A. Rajabpour, F. Kowsary, Temperature profile for nanoscale Poiseuille flow: a multiscale study, J. Mech. Sci. Technol. 30 (2016) 803-807. doi:10.1007/s12206-0150929-9.

[23] Z. Shi, M. Barisik, A. Beskok, Molecular dynamics modeling of thermal resistance at argon-graphite and argon-silver interfaces, Int. J. Therm. Sci. 59 (2012) 29-37. doi:10.1016/j.ijthermalsci.2012.04.009.

[24] A.T. Pham, M. Barisik, B.H. Kim, Interfacial thermal resistance between the graphenecoated copper and liquid water, Int. J. Heat Mass Transf. 97 (2016) 422-431. doi:10.1016/j.ijheatmasstransfer.2016.02.040.

[25] A. Amani, S.M.H. Karimian, M. Seyednia, A molecular dynamics simulation on the effect of different parameters on thermal resistance of graphene-argon interface, Mol. Simul. 43 (2017) 276-283. doi:10.1080/08927022.2016.1265959.

[26] T.Q. Vo, B.H. Kim, Interface thermal resistance between liquid water and various metallic 
surfaces, Int. J. Precis. Eng. Manuf. 16 (2015) 1341-1346. doi:10.1007/s12541-015-01760 .

[27] M. Barisik, A. Beskok, Temperature dependence of thermal resistance at the water/silicon interface, Int. J. Therm. Sci. 77 (2014) 47-54. doi:10.1016/j.ijthermalsci.2013.10.012.

[28] J. Ghorbanian, A. Beskok, Temperature profiles and heat fluxes observed in molecular dynamics simulations of force-driven liquid flows, Phys. Chem. Chem. Phys. 19 (2017) 10317-10325. doi:10.1039/C7CP01061C.

[29] Y. Tang, T. Fu, Y. Mao, Y. Zhang, W. Yuan, Molecule Dynamics Simulation of Heat Transfer Between Argon Flow and Parallel Copper Plates, J. Nanotechnol. Eng. Med. 5 (2014) 034501. doi:10.1115/1.4029158.

[30] J. Vera, Y. Bayazitoglu, Temperature and heat flux dependence of thermal resistance of water/metal nanoparticle interfaces at sub-boiling temperatures, Int. J. Heat Mass Transf. 86 (2015) 433-442. doi:10.1016/j.ijheatmasstransfer.2015.02.033.

[31] Q. Li, C. Liu, Molecular dynamics simulation of heat transfer with effects of fluid-lattice interactions, Int. J. Heat Mass Transf. 55 (2012) 8088-8092. doi:10.1016/j.ijheatmasstransfer.2012.08.045.

[32] M. Morciano, M. Fasano, A. Nold, C. Braga, P. Yatsyshin, D.N. Sibley, B.D. Goddard, E. Chiavazzo, P. Asinari, S. Kalliadasis, Nonequilibrium molecular dynamics simulations of nanoconfined fluids at solid-liquid interfaces, J. Chem. Phys. 146 (2017) 244507. doi:10.1063/1.4986904.

[33] Y. Chen, C. Zhang, Role of surface roughness on thermal conductance at liquid-solid interfaces, Int. J. Heat Mass Transf. 78 (2014) 624-629. doi:10.1016/J.IJHEATMASSTRANSFER.2014.07.005.

[34] A.T. Pham, M. Barisik, B. Kim, Molecular dynamics simulations of Kapitza length for argon-silicon and water-silicon interfaces, Int. J. Precis. Eng. Manuf. 15 (2014) 323-329. doi:10.1007/s12541-014-0341-X.

[35] F. Faraji, A. Rajabpour, Fluid heating in a nano-scale Poiseuille flow: A non-equilibrium molecular dynamics study, Curr. Appl. Phys. 17 (2017) 1646-1654. doi:10.1016/j.cap.2017.09.008.

[36] B.Y. Cao, J. Sun, M. Chen, Z.Y. Guo, Molecular momentum transport at fluid-solid interfaces in MEMS/NEMS: A review, Multidisciplinary Digital Publishing Institute (MDPI), 2009. doi:10.3390/ijms10114638.

[37] R. Rabani, G. Heidarinejad, J. Harting, E. Shirani, Interplay of confinement and density on the heat transfer characteristics of nanoscale-confined gas, Int. J. Heat Mass Transf. 126 (2018) 331-341. doi:10.1016/j.ijheatmasstransfer.2018.05.028.

[38] S. Plimpton, Fast Parallel Algorithms for Short-Range Molecular Dynamics, J. Comput. Phys. 117 (1995) 1-19. doi:10.1006/jcph.1995.1039.

[39] M.P. Allen, D.J. Tildesley, J.R. Banavar, Computer Simulation of Liquids, Phys. Today. 42 (1989) 105-106. doi:10.1063/1.2810937.

[40] B.H. Kim, A. Beskok, T. Cagin, Thermal interactions in nanoscale fluid flow: Molecular dynamics simulations with solid-liquid interfaces, Microfluid. Nanofluidics. 5 (2008) 551-559. doi:10.1007/s10404-008-0267-7.

[41] B.H. Kim, A. Beskok, T. Cagin, Viscous heating in nanoscale shear driven liquid flows, Microfluid. Nanofluidics. 9 (2010) 31-40. doi:10.1007/s 10404-009-0515-5.

[42] N. Asproulis, D. Drikakis, Boundary slip dependency on surface stiffness, Phys. Rev. E Stat. Nonlinear, Soft Matter Phys. 81 (2010) 1-5. doi:10.1103/PhysRevE.81.061503. 
[43] J.H. Irving, J.G. Kirkwood, The Statistical Mechanical Theory of Transport Processes. IV. The Equations of Hydrodynamics, J. Chem. Phys. 18 (1950) 817-829. doi: $10.1063 / 1.1747782$.

[44] B.D. Todd, P.J. Daivis, D.J. Evans, Heat flux vector in highly inhomogeneous nonequilibrium fluids, Phys. Rev. E. 51 (1995) 4362-4368. doi:10.1103/PhysRevE.51.4362.

[45] G. Karniadakis, A. Beskok, N. Aluru, Microflows and Nanoflows: Fundamentals and Simulation, Springer Science $\{\&\}$ Business Media, 2006. http://books.google.com/books?hl=en\%7B \&\%7Dlr=\%7B\&\%7Did=vDiLnJHSqvYC\%7B $\& \% 7$ Dpgis $=1$.

[46] E.W. Lemmon, M.O. McLinden, D.G. Friend, P. Linstrom, W. Mallard, NIST chemistry WebBook, NIST standard reference database number 69, Natl. Inst. Stand. Technol. Gaithersbg. (2011). 